\title{
Propuesta metodológica para el cálculo de los derechos de pesca e implicancias de política pesquera: el caso de la anchoveta peruana
}

(Engraulis ringens)

\begin{abstract}
Methodological proposal for the calculation of fishing fees and fisheries policy implications: the case of the Peruvian anchovy
\end{abstract}

(Engraulis ringens)

Recibido: 04 de junio de 2020

Aprobado: 25 de junio de 2020

Autor:

Julio César

Ruiz Ramírez

Ing. Pesquero

Universidad Nacional

Agraria La Molina, Perú

Estudios de Doctorado

en Gobierno y Políticas

Públicas del Instituto

de Gobierno y de

Gestión Pública

Correo:

juliocesar.ruiz13@yaho o.com.pe

\section{RESUMEN}

El presente estudio tiene como objetivo demostrar la existencia de un marco teórico que permita sustentar -desde una perspectiva científica- una metodología para el cálculo de los derechos de pesca de la anchoveta peruana. Se utilizó para este efecto, el modelo bioeconómico de optimización dinámica en tiempo continuo de Clark-Munro (1975), cuyos resultados se validaron con el modelo bioeconómico estático de Gordon-Schaefer (1954), ampliamente usado en la economía pesquera. El modelo de Clark-Munro se sustenta en la teoría del capital, la teoría de la inversión y la teoría del control óptimo. Se evalúa su aplicación en series de datos de pesca de la zona norte-centro correspondientes al período 2004-2008, previo a la reforma de ordenación pesquera de la anchoveta, y al período 2009-2017 correspondiente a la reforma de ordenación pesquera de la anchoveta. Las series de datos de captura y de esfuerzo, se obtuvieron del "Programa de Vigilancia y Control de la Pesca y Desembarque en el Ámbito Marítimo", a cargo de empresas privadas de inspección seleccionadas por la administración pesquera. El número total de embarcaciones anchoveteras de la zona de pesca se dividió en tres grupos y se estandarizó el esfuerzo pesquero y la captura por unidad de esfuerzo (CPUE). La medida de esfuerzo pesquero seleccionada, resultó compatible sólo con el período de pre-reforma 2004-2008, obteniéndose los parámetros del rendimiento máximo sostenible (MSY), mediante el método de Verhulst- Schaefer; los parámetros del rendimiento máximo económico (MEY), a través del método de Gordon-Schaefer; y los parámetros del rendimiento óptimo sostenible (OSY), por el método de Clark-Munro. Por último, los derechos de pesca se calcularon a partir de la renta del recurso óptima en el nivel de OSY estimada mediante el método de Clark-Munro.

Palabras clave: anchoveta, derechos de pesca, CPUE, modelo bioeconómico dinámico, renta del recurso.

\section{ABSTRACT}

The present study aims to demonstrate the existence of a theoretical framework that would allow to sustain - from a scientific perspective - a methodology for the calculation of fishing fees for the Peruvian anchovy. For this purpose, the bioeconomic model of continuous time dynamic optimization by Clark-Munro (1975) was used, whose results were validated with the static bioeconomic model by Gordon-Schaefer (1954), widely used in the fisheries economics. The model of Clark-Munro (1975) is based on the theory of capital, the theory of investment and 
the theory of optimal control. Its application in fisheries data series for the northcentral zone of the coast corresponding to the period 2004-2008, prior to the reform of anchovy fishery management, and to the period 2009-2017 corresponding to the reform of anchovy fishery management, is evaluated. The series of catch and fishing effort data, were obtained from the "Program of Surveillance and Control of Fishing and Landing in the Maritime Area", in charge of private inspection companies selected by the fisheries administration. The total number of anchovy vessels in the fishing zone was divided into three groups and fishing effort and catch per unit of effort (CPUE) were standardized. The selected fishing effort measure was compatible only with the pre-reform period 2004-2008, obtaining the maximum sustainable yield (MSY) parameters, using the Verhulst-Schaefer method; the maximum economic yield (MEY) parameters, through the Gordon-Schaefer method; and the optimal sustainable yield (OSY) parameters, by the Clark-Munro method. Finally, the fishing fees were calculated from the optimal rent of the resource at the OSY level estimated by the Clark-Munro method.

Key words: anchovy, fishing fees, CPUE, dynamic bioeconomic model, resource rent.

\section{INTRODUCCIÓN}

El derecho de pesca es el monto que los armadores de embarcaciones pesqueras de mayor escala y de menor escala, están obligados a pagar al Estado por extraer recursos hidrobiológicos que constituyen patrimonio de la Nación, según la Ley General de Pesca.

La forma y modo de cálculo de los derechos de pesca del recurso anchoveta, ha sufrido cambios a lo largo del tiempo. Tradicionalmente, el derecho de pesca de este recurso, se estableció como un porcentaje de la Unidad Impositiva Tributaria (UIT), pero a partir del año 2006 empezó a aplicarse el valor FOB de la harina de pescado como unidad referencial, debido a la tendencia creciente del precio mundial de la harina de pescado, producto principal del procesamiento de este pequeño recurso pelágico. El derecho de pesca es un concepto asociado directamente con la actividad extractiva; sin embargo, como podrá advertirse, ni la UIT ni el valor FOB, tienen vinculación directa con la actividad extractiva del recurso.

Los derechos de pesca han sido conceptualizados de diversas maneras en las pesquerías del mundo. En algunos países, es un impuesto o corresponde a una licencia de pesca; en otros, el concepto de base es la renta del recurso (Galarza y Collado, 2013).

Varios autores han definido el término "renta del recurso". Rogers y Webster (2007), se refieren a la renta del recurso como el exceso de beneficios o beneficio "supernormal" sobre el nivel ganado en un mercado competitivo. La renta del recurso, afirman, es igual a la diferencia entre los ingresos y los costos (incluyendo un rendimiento competitivo del capital). Bjørndal y Bezabih (2010), precisan que el costo incluye la depreciación y un retorno normal del capital. Y, Wessel (1967), citado en Campbell y Haynes (1990), es bastante claro cuando sostiene que la renta del recurso refleja los excedentes después de haberse recuperado todos los costos, incluyendo la necesaria rentabilidad del capital que justifica la inversión.

Si la renta del recurso es la sobreutilidad o beneficio neto adicional al retorno normal del capital invertido, cabe preguntarse, entonces, a quién pertenecería dicho beneficio. Gordon (1954), pionero de la economía pesquera, despeja esta cuestión, al afirmar que "en la pesquería marina, el recurso natural no es propiedad privada; por lo tanto, nadie puede apropiarse de la renta que pueda generar."

En consecuencia, desde una perspectiva teórica, la renta del recurso pertenecería al Estado, en su calidad de propietario del recurso, cuyo monto podría considerarse como el derecho de pesca que el armador pesquero se obliga a pagar al Estado.

En tal sentido, cabe preguntarse si la renta del recurso puede constituirse en la base teórica para el cálculo del derecho de pesca del recurso anchoveta. De hecho, Galarza y Collado (2013), estimaron los derechos de pesca de la anchoveta peruana a partir de la renta del recurso determinada mediante el modelo bioeconómico estático de Gordon-Schaefer, pero recomendaron aplicar un modelo de optimización dinámica en tiempo continuo.

Para tal motivo, se estimó conveniente aplicar en este estudio el modelo bioeconómico de optimización dinámica en tiempo continuo de Clark y Munro (1975), el cual maximiza la renta del recurso. Su herramental teórico está constituido por la teoría del capital, la teoría de la inversión y la teoría del control óptimo basada en el principio del máximo de Pontryagin. Clark y Munro 
(1975), desarrollaron en su modelo las viejas ideas de Anthony Scott (1955) uno de los pioneros de la economía de los recursos naturales explicitadas en su libro Natural Resources Economics: The Economics of Conservation, donde deja sentado por primera vez y en forma "absolutamente clara que la economía de los recursos naturales es, primero y ante todo, una aplicación de las teorías del capital y de la inversión de los economistas" (Clark y Munro, 2017).

El modelo matemático de Clark-Munro, es una formulación dinámica predictiva que aplica la "teoría del capital que trata sobre los stocks y aborda la cuestión de cuál es el stock de capital óptimo de un tipo particular de stock; la teoría de la inversión que trata sobre los flujos y aborda la cuestión de cuál es la tasa óptima a la que debe aumentarse el stock de capital, o disminuirse, si el stock actual está por debajo o por encima del nivel de stock óptimo" (Ackley, 1978; citado en Clark y Munro, 2017), y la teoría del control óptimo que maximiza la renta del recurso.

\section{Breve revisión sobre los derechos de pesca}

La Ley General de Pesca y su reglamento establecen que los armadores de embarcaciones pesqueras de mayor escala (más de $32,6 \mathrm{~m} 3$ de capacidad de bodega) y de menor escala (menos de 32, 6 m3 de capacidad de bodega) están obligados a pagar un monto por el derecho a extraer recursos pesqueros que son de propiedad pública. A este monto se le denomina derecho de pesca; y es el pago que hace el armador pesquero al Estado por cada tonelada de anchoveta desembarcada en las plantas de procesamiento de harina de pescado.

A lo largo del tiempo, la forma del cálculo de los derechos de pesca del recurso anchoveta ha experimentado sucesivas modificaciones, tal como se muestra en la Tabla 1. Inicialmente, el monto de pago del derecho de pesca se estableció como un porcentaje de la Unidad Impositiva Tributaria (UIT); sin embargo, cuando el precio mundial de la harina de pescado empezó a registrar una tendencia alcista, se modificó su modo de cálculo por el valor FOB de la harina de pescado. Pero, ambos parámetros no guardan relación directa con la actividad extractiva.

Tabla 1. Cambios normativos relativos a los derechos de pesca de la anchoveta

\begin{tabular}{|c|c|}
\hline Norma & Derecho de pesca \\
\hline $\begin{array}{l}\text { Reglamento de la Ley General de Pesca (DS N } \\
\text { 012-2001-PE) }\end{array}$ & $\begin{array}{l}0.075 \% \text { UIT/tonelada métrica extraída de los recursos } \\
\text { anchoveta, sardina, jurel y caballa }\end{array}$ \\
\hline $\begin{array}{l}\text { D.S. } N^{\circ} 007-2002-\text { PRODUCE } \\
\text { (Publicado el 05/12/2002) }\end{array}$ & $\begin{array}{l}0.23 \% \text { UIT/tonelada métrica extraída de los recursos } \\
\text { hidrobiológicos destinados al consumo humano indirecto. }\end{array}$ \\
\hline $\begin{array}{l}\text { D.S. } N^{\circ} 011-2002-\text { PRODUCE } \\
\text { (Publicado el } 20 / 12 / 2002 \text { ) }\end{array}$ & $\begin{array}{l}0.116 \% \text { UIT/tonelada métrica extraída de los recursos } \\
\text { hidrobiológicos destinados al consumo humano indirecto. }\end{array}$ \\
\hline $\begin{array}{l}\text { D.S. N॰024-2006-PRODUCE } \\
\text { (Publicado el } 26 / 11 / 2006 \text { ) }\end{array}$ & $\begin{array}{l}\text { El pago de derechos por extracción de recursos hidrobiológicos } \\
\text { destinados al consumo humano indirecto, se efectuará sobre la } \\
\text { base de aplicar el } 0.25 \% \text { del valor FOB por tonelada de harina } \\
\text { de pescado, computable sobre el precio promedio mensual } \\
\text { según información oficial }\end{array}$ \\
\hline $\begin{array}{l}\text { D. Legislativo } \mathrm{N}^{\circ} \text { 1084. Primera Disposición } \\
\text { Final. (Publicado el 28/06/2008) }\end{array}$ & $\begin{array}{l}\text { Durante el período de vigencia del aporte social obligatorio se } \\
\text { mantendrá sin alteración el monto y la forma de cálculo de los } \\
\text { Derechos de Pesca por extracción de recursos hidrobiológicos } \\
\text { destinados al Consumo Humano Indirecto, aprobados mediante } \\
\text { el DS № 024-2006-PRODUCE. }\end{array}$ \\
\hline
\end{tabular}

Fuente: Galarza y Collado (2013)

Mediante la Primera Disposición Final del Decreto Legislativo 1084, publicado el 2008, se estableció el congelamiento del monto del derecho de pesca de la anchoveta por 10 años, a cambio de que los industriales pesqueros asumieran determinados aportes económicos adicionales destinados a resolver la problemática del personal excedente y de los pescadores jubilados, así como el compromiso de financiamiento del programa de vigilancia y control de la extracción del recurso anchoveta. 
Tabla 2. Derechos de pesca recaudados por extracción de anchoveta (2012 - 2016)

\begin{tabular}{|c|c|c|c|c|c|c|c|}
\hline Detalle & 2012 & 2013 & 2014 & 2015 & 2016 & Promedio & Porcentaje \\
\hline E/P acero naval & $25,380,614$ & $44,641,499$ & $19,677,087$ & $44,530,892$ & $27,877,217$ & $32,421,462$ & 82 \\
\hline $\mathrm{E} / \mathrm{P}$ madera & $5,281,456$ & $10,382,388$ & $3,668,396$ & $9,225,571$ & $7,629,494$ & $7,237,461$ & 18 \\
\hline Total (Nuevos Soles) & $30,662,070$ & $55,023,887$ & $23,345,483$ & $53,756,463$ & $35,506,711$ & $39,658,923$ & 100 \\
\hline Desembarque & $3,615.5$ & $4,754.1$ & $2,255.4$ & $3,686.8$ & $2,732.7$ & \multirow{4}{*}{$\begin{array}{r}11.60 \\
3.91\end{array}$} & \\
\hline Soles/TM & 8.48 & 11.57 & 10.35 & 14.58 & 12.99 & & \\
\hline \multirow{2}{*}{$\begin{array}{l}\text { US } \$ / T M \\
\text { Tipo de cambio } \\
\text { promedio anual }\end{array}$} & 3.21 & 4.29 & 3.64 & 4.57 & 3.84 & & \\
\hline & 2.64 & 2.7 & 2.84 & 3.19 & 3.38 & & \\
\hline
\end{tabular}

Fuente: Ministerio de la Producción y Banco Central de Reserva

Elaboración propia

La Tabla 2 muestra información oficial proporcionada por el Ministerio de la Producción, sobre recaudación de derechos de pesca del recurso anchoveta y volumen de desembarque de dicho recurso en todo el litoral, correspondiente al período 2012-2016. A partir de dicha información, se determinó el monto promedio anual del derecho de pesca por tonelada métrica en nuevos soles (S/. 11,60/TM) y en dólares (US\$ 3,91/TM) tomando en cuenta el tipo de cambio promedio anual calculado por el Banco Central de Reserva (BCR).

No cabe duda que el cambio de método del cálculo de los derechos de pesca para el recurso anchoveta significó un avance, tal como se muestra en la Figura 2, pero insuficiente. El año 2006, el Estado apenas cobraba a los armadores US\$1,1/TM por concepto de derecho de pesca de anchoveta. El valor más alto se obtuvo el año 2015: US\$ 4,5/TM, aplicando el valor FOB por tonelada de harina de pescado.

Figura 2. Evolución del valor del derecho de pesca de anchoveta US\$/TM

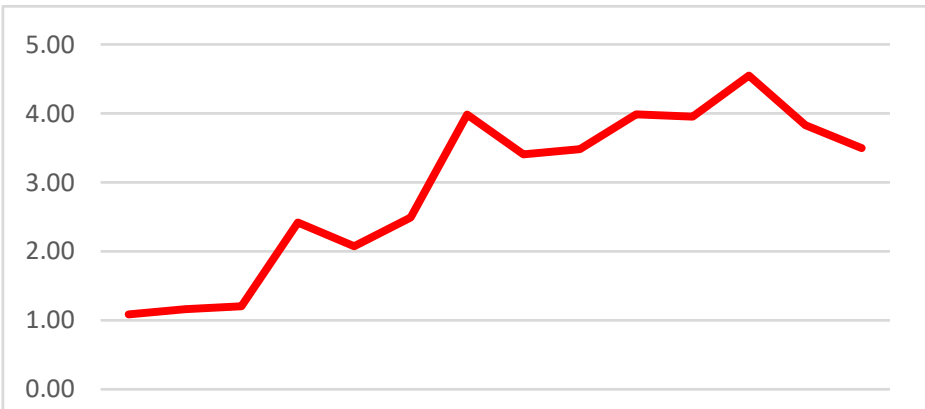

20042005200620072008200920102011201220132014201520162017

Fuente: MEF y ADUANET

Elaboración propia

\section{Justificación de la investigación}

De La Puente et al. (2011), afirman que, el monto pagado por derechos de pesca no es proporcional a los costos de operación de la industria, al precio de la harina y el aceite de pescado o a la rentabilidad de las empresas. Asimismo, señalan que esta situación de desequilibrio entre los costos de operación y los derechos de pesca tiene efectos negativos graves para la recaudación de fondos por parte del Estado.

Paredes (2012), propone una metodología para determinar los derechos de pesca en la pesquería de anchoveta, a partir de los ingresos y costos derivados de su procesamiento en harina y aceite de pescado, con el fin de corregir la ínfima tasa del derecho de pesca en la pesquería de la anchoveta (US\$ 3,38 por TM de anchoveta desembarcada). Bajo este esquema metodológico, y según la utilidad del año 2011, determina un monto de US\$182,09 por TM de anchoveta desembarcada que debería cobrarse como derecho de pesca.

Sin embargo, conviene anotar que si bien existe una integración patrimonial entre la actividad extractiva y la de procesamiento del recurso, el derecho de pesca debiera estar en 
correspondencia únicamente con la renta del recurso, es decir, con la utilidad del armador pesquero.

Galarza y Collado (2013), señalan que "el derecho de pesca, tal cual se aplica en la pesquería de la anchoveta, no guarda relación alguna con el costo de uso del recurso o con lo que se podría llamar la regalía que cobra el Estado. El derecho de pesca debería ser calculado sobre la base de la renta del recurso, es decir, sobre la diferencia entre los ingresos totales y los costos totales".

Clark (2006), sostiene que los sistemas de cuotas individuales pueden mejorar el rendimiento económico y biológico de una pesquería, pero también plantean varios problemas importantes, entre ellos, el enriquecimiento de unos pocos seleccionados y la concentración de la propiedad de la cuota, lo cual es el resultado directo de la falta de captación de una porción sustancial de la renta del recurso para la cartera pública, es decir, el cobro de un monto apropiado por concepto de derechos de pesca. Añade que "dividir la torta puede no ser fácil, pero asumir que el público no tiene derecho a él no es un resultado justo". También sostiene que "el gobierno puede retener una parte de la renta del recurso al cobrar regalías de desembarque, como en otras industrias de recursos. Exactamente qué parte de la renta (más allá de los costos de gestión) debe recaudar el Estado es una cuestión de política, que la industria pesquera intentará sesgar a su favor. Otro método para resolver el problema es a través de subastas de cuotas de duración limitada".

Un estudio privado de la firma Apoyo Consultoría de 2016, sobre los derechos de pesca en Perú y Chile, confirma el monto promedio anual de US\$3,9/TM pagado por los armadores anchoveteros de Perú y, asimismo, reporta el monto de derecho de pesca pagado en Chile, equivalente a US\$11,2/TM por la extracción de los recursos destinados a la elaboración de harina de pescado, que está dado por la suma de la Patente de Beneficio Fiscal y el Impuesto Específico por Especie, tal como se muestra en la Figura 1. Cabe indicar que, según la Ley General de Pesca y Acuicultura de Chile, la patente se paga en marzo, de acuerdo al tamaño de la embarcación, y el impuesto, en julio según la cuota de captura. En Perú, el derecho de pesca está dado por un único concepto, mientras que en Chile está constituido por dos conceptos.

\section{Figura 1. Derechos de Pesca en Perú y Chile 2016 (US\$/TM)}

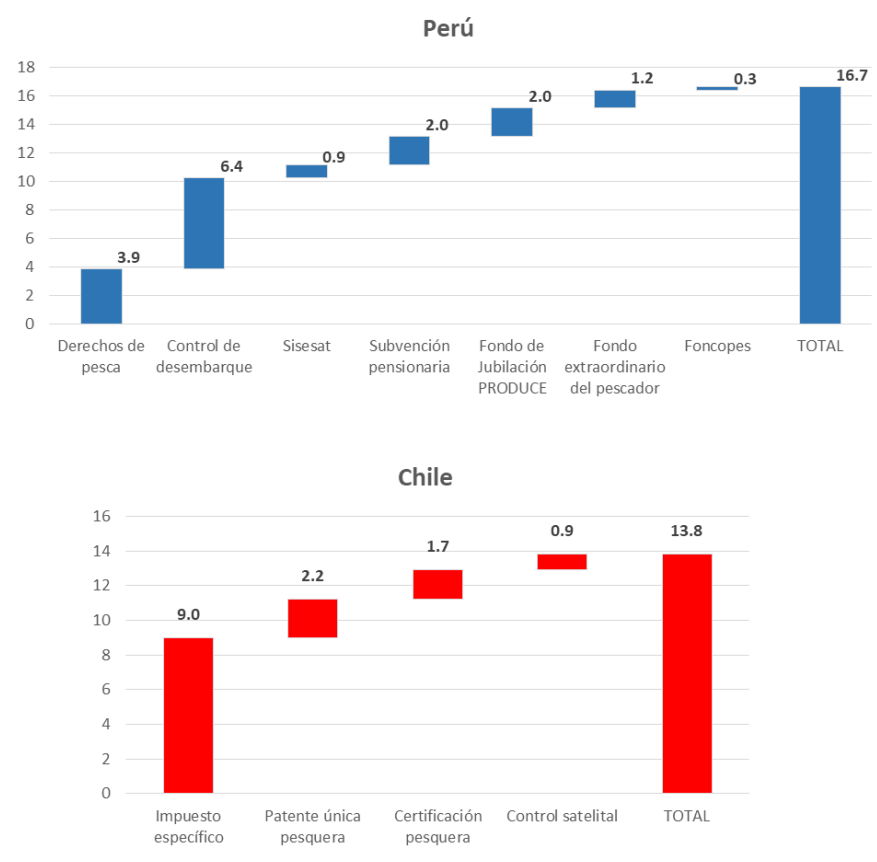

Fuente: SUNAT, PRODUCE, Servicio de Impuestos Internos de Chile y Subsecretaría de Pesca y Acuicultura de Chile.

Elaboración: APOYO Consultoría.

Csirke y Gumy (1996), recomiendan que en el cálculo del derecho de pesca, se tenga en cuenta el margen de rentabilidad y beneficios potenciales a que accede el armador pesquero y el derecho que tiene la comunidad y la Nación a participar de las rentas y beneficios producidos por la explotación de un bien común, así como la necesidad de sufragar los gastos en que incurre el Estado para garantizar la conservación y explotación racional de dicho bien común, incluidos 
los costos de investigación y los mecanismos de monitoreo, control y vigilancia, necesarios para una adecuada ordenación y planificación del desarrollo de la pesquería en cuestión.

Consecuentemente, para determinar si el monto pagado por derecho de pesca es razonable, se requiere conocer la renta del recurso (margen de rentabilidad) a partir de los niveles de ingresos y costos de la industria pesquera extractiva en general, los cuales dependen ciertamente de variables biológicas y económicas. Precisamente, la academia ha generado modelos matemáticos en el marco de la teoría bioeconómica, orientados a resolver este tipo de problemas, que el presente estudio recoge con el fin de analizar la factibilidad de su aplicación.

Por las consideraciones expuestas, se estima necesario efectuar un estudio de la renta pesquera de la anchoveta, basada en un modelo bioeconómico dinámico en tiempo continuo, que pudiera utilizarse como fundamento básico para el cálculo de los derechos de pesca del recurso anchoveta.

Para el efecto, se tomará como base el modelo bioeconómico dinámico en tiempo continuo, formulado por Clark y Munro (1975), que transforma la teoría económica pesquera en términos de optimización estática que fundó Gordon (1954), en una de optimización dinámica en tiempo continuo, aplicando los fundamentos básicos de la teoría moderna del capital, de la teoría de la inversión y de la teoría del control óptimo.

Clark y Munro (1975), determinan la solución matemática que establece el nivel de stock óptimo del recurso que genera un rendimiento óptimo de captura en el tiempo, garantizando el desarrollo de una pesquería económica y biológicamente sostenible. Es decir, crean una herramienta de administración pesquera eficiente.

El modelo se sustenta en el empleo de las variables: captura, esfuerzo de pesca, precio del recurso y costo del esfuerzo. Adicionalmente, el modelo utiliza la tasa social de descuento que se aplica a los proyectos de inversión pública.

Según la literatura bioeconómica, el citado modelo bioeconómico, se constituye en una importante herramienta de gestión pesquera que contribuye a asegurar la explotación sostenible de los recursos pesqueros, pues permite estimar, en primer término, el nivel óptimo de biomasa y a partir de aquella, calcular el nivel óptimo de captura del recurso (Flaaten, 2014; Viet Thanh, 2006; Berachi, 2003). Y, se sustenta en un sólido marco teórico basado en la teoría del capital, la teoría de la inversión y la teoría del control óptimo; esta última basada en el principio del máximo de Pontryagin (Munro y Sumaila, 2015).

A partir de este herramental matemático, el presente trabajo de investigación pretende analizar la aplicación de la renta del recurso como base para la formulación del cálculo de los derechos de pesca del recurso anchoveta, con el propósito de plantear una propuesta metodológica con el sustento técnico necesario que permita contribuir a una gestión más eficiente de la pesquería de la anchoveta, no sólo en términos del establecimiento de una retribución equitativa al Estado como derecho de pesca por la extracción del recurso, sino, de la aplicación de una política de captura óptima a partir de su correspondiente nivel de biomasa óptima del recurso.

\section{Objetivo de la investigación}

Evaluar en qué medida la renta del recurso, estimada mediante el modelo bioeconómico de optimización dinámica en tiempo continuo de Clark y Munro (1975), puede constituirse en un fundamento económico válido para el cálculo de los derechos de pesca de la anchoveta peruana (Engraulis ringens).

\section{Marco teórico}

El modelo bioeconómico de optimización dinámica de Clark-Munro (1975), se basa en el conocido modelo biológico de Schaefer (1954), denominado también modelo Verhulst-Schaefer, y en el modelo bioeconómico estático de Gordon (1954), conocido ampliamente en la economía pesquera como modelo Gordon-Schaefer. Por tanto, resulta conveniente describir previamente ambos modelos matemáticos, cuyos resultados servirán, además, de marco referencial para la aplicación del modelo Clark-Munro. 


\section{a) Modelo biológico de Schaefer}

Schaefer (1954), desarrolló un modelo de equilibrio pesquero basado en el modelo de crecimiento poblacional de Verhulst ${ }^{1}$, mediante el cual estima el nivel de Rendimiento Máximo Sostenible (MSY) ${ }^{2}$ de cada stock (población) de atún. En este modelo se asume que la biomasa sigue una función de crecimiento logístico (Berachi, 2003), la cual es una representación matemática del crecimiento de la biomasa de un stock animal, que describe una curva de crecimiento natural simétrica en forma de campana (Flaaten, 2014), tal como se muestra en la Figura 3.

\section{Figura 3. Rendimiento Máximo Sostenible (MSY)}

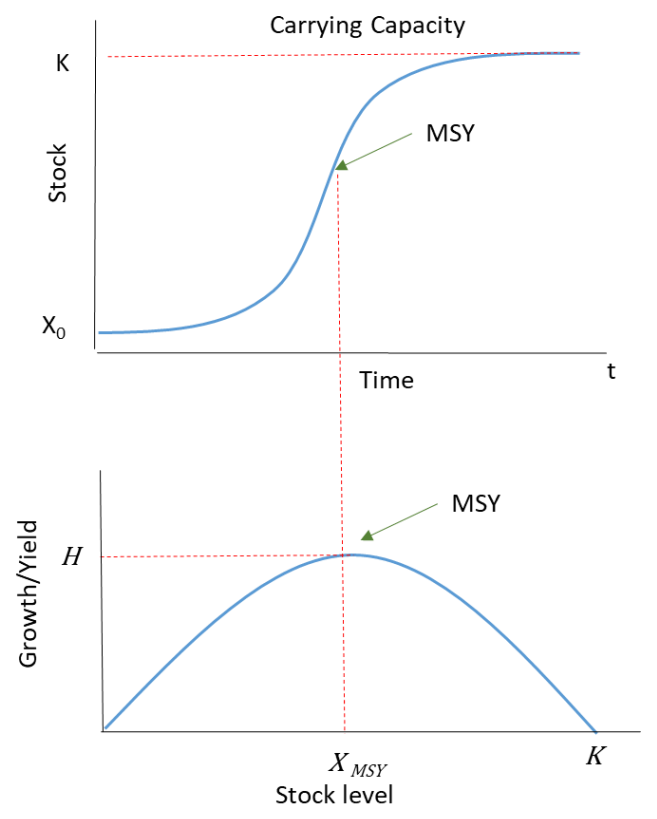

El modelo biológico de Verhulst-Schaefer (1954), considera el cambio de la biomasa $(X)$ por unidad de tiempo, en situación de pesca, como sigue:

$$
\frac{d X}{d t}=F(X)-H
$$

donde $F(X)$ es el crecimiento natural de la biomasa y $H$, la captura. $F(X)$ es una función que se basa en una ecuación de crecimiento natural de tipo logístico, que sigue una curva parabólica 0 logística simétrica:

$$
F(X)=r X(1-X / K)
$$

"Dicha ecuación fue diseñada y discutida primero por P. F. Verhulst (1838) y más tarde descubierta por R. Pearl (1925). El parámetro $r$ es la tasa máxima de crecimiento relativo, también llamada tasa intrínseca de crecimiento y $K$ es la capacidad de carga del hábitat, ambos parámetros se asumen fijos" (Flaaten, 2014).

Con relación a la ecuación (1), el modelo biológico de Verhulst-Schaefer (1954) considera la siguiente función de captura $H$, siendo esta formulación el aporte sustantivo del modelo:

$$
H=q E X
$$

\footnotetext{
${ }^{1}$ Matemático Belga (1804-1849)

${ }^{2}$ Maximum Sustainable Yield
} 
Según Berachi (2003), esta función asume que el esfuerzo pesquero, siempre remueve una proporción constante de la biomasa. $E=$ esfuerzo de pesca y $q$ es un coeficiente de capturabilidad constante. $Y$, el rendimiento de equilibrio ocurre cuando la tasa de captura es igual a la tasa de crecimiento natural, es decir, cuando la tasa de cambio de la biomasa $d X / d t=F(X)-H(E, X)=0$; esto es, $H(E, X)=F(X)$. Entonces, teniendo en cuenta (2) y (3), se genera la ecuación de equilibrio:

$$
q E X=r X\left(1-\frac{X}{K}\right)
$$

A partir de (4) se puede obtener la biomasa $X$ que genera el rendimiento de equilibrio:

$$
X=K\left(1-\frac{q}{r} E\right)
$$

Insertando (5) en la ecuación (3), da lugar a:

$$
\begin{aligned}
& H=q E K\left(1-\frac{q}{r} E\right) \\
& H=q K E-\frac{q^{2} K}{r} E^{2}
\end{aligned}
$$

La ecuación (7) es una función de captura a largo plazo, cuyos parámetros pueden estimarse a partir de los datos de captura y esfuerzo pesquero a través de una regresión lineal si hacemos: $q K=a$ y $q^{2} K / r=b$; con lo cual la ecuación (7) queda como sigue:

$$
H=a E-b E^{2}
$$

Entonces, la regresión lineal puede realizarse convirtiendo la ecuación (8) en:

$$
\frac{H}{E}=a-b E
$$

Con los datos de captura y esfuerzo pesquero disponibles, se puede estimar los parámetros $a$ y $b$ a través de un modelo de regresión lineal simple, considerando la relación de dependencia entre la variable dependiente "captura por unidad de esfuerzo" (CPUE) y la variable independiente "esfuerzo". La CPUE y el esfuerzo siguen una relación uniformemente lineal, con pendiente negativa, tal como se muestra en la Figura 4:

Figura 4. Relación Captura por Unidad

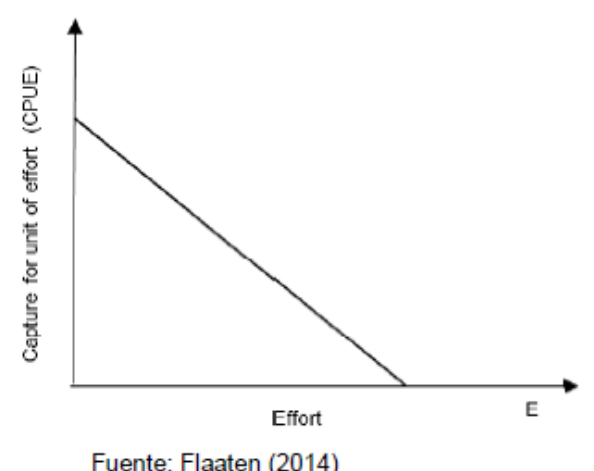

Fuente: Flaaten (2014) 


\section{de Esfuerzo (CPUE) y Esfuerzo}

El esfuerzo en el Rendimiento Máximo Sostenible $\left(E_{M S Y}\right)^{3}$ puede estimarse maximizando la ecuación (8), esto es, tomando la derivada parcial de $H$ con respecto a $E$; e igualando a cero:

$$
E_{M S Y}=\frac{\mathrm{a}}{2 \mathrm{~b}}
$$

Sustituyendo luego (10) en la ecuación (8) se obtiene el Rendimiento Máximo Sostenible $\left(H_{M S Y}\right)^{4}$ o captura máxima sostenible:

$$
H_{M S Y}=\frac{\mathrm{a}^{2}}{4 \mathrm{~b}}
$$

Para determinar el nivel de biomasa $X_{M S Y}$, correspondiente al Rendimiento Máximo Sostenible, se reemplaza (10) en la ecuación (12), que resulta de igualar [3] y [8]:

$$
q E X=a E-b E^{2}
$$

lo cual permite obtener $X_{M S Y^{5}}$, como:

$$
X_{M S Y}=\frac{\mathrm{a}}{2 \mathrm{q}}
$$

En consecuencia, el modelo biológico de Verhulst-Schaefer, permite determinar si una pesquería se viene desarrollando en forma sostenible, a partir del análisis de la serie de datos de captura y esfuerzo pesquero, lo cual conllevaría, en su caso, a adoptar decisiones de política pesquera orientadas a superar la problemática que se pudiera advertir.

\section{b) Modelo bioeconómico estático de Gordon-Schaefer}

Este modelo se basa en la integración del modelo biológico de Verhulst-Schaefer (1954) y el modelo bioeconómico de Gordon (1954), realizada por Schaefer (1957). Sin embargo, Munro y Sumaila (2015), sostienen que "el modelo económico de Gordon (1954) se basa firmemente en el fundamento del modelo biológico desarrollado por M. B. Schaefer. El vínculo es tan cercano que el modelo se conoce comúnmente en la literatura como el modelo de Gordon-Schaefer." 6

Gordon (1954), en la fundamentación de su teoría económica, sostiene que el término "gestión pesquera" focaliza su atención en la captura máxima sostenible, la cual, desde su punto de vista, tiene serias limitaciones en el tratamiento del problema [de la sobreexplotación] de la pesca, dado que, al poner la atención en la maximización de la captura, descuida por completo los aportes de otros factores de producción que se utilizan en la pesca como los costos, y que

\footnotetext{
${ }^{3}$ También puede expresarse como $(\mathrm{r} / \mathrm{q}) / 2$

${ }^{4}$ También puede expresarse como $r K / 4$.

${ }^{5}$ Equivale a $K / 2$

${ }^{6}$ Esto no parece ser exacto, pues ambos investigadores publicaron sus artículos fundamentales el mismo año; además, Gordon (1954) no menciona a Schaefer (1954) en sus fuentes de referencia. Lo que sí se puede verificar es que ambos modelos se encuentran integrados en el estudio de Schaefer (1957), en cuya bibliografía se cita el artículo del economista canadiense. No obstante, no deja de sorprender que ambos investigadores coincidan en la misma ecuación de captura/desembarque.
} 
deben ser considerados. Por eso, denomina su teoría como "teoría de la utilización óptima de los recursos pesqueros", expresada en la maximización del rendimiento económico neto: la diferencia entre costo total, por un lado y el ingreso total, por el otro.

Schaefer (1957), integró en uno solo, el modelo biológico de Schaefer (1954) y el modelo bioeconómico de Gordon (1954), conocido ampliamente como modelo económico estático de Gordon-Schaefer, en el cual, la renta económica total de la pesquería está dada por la siguiente ecuación:

$$
\pi(E)=T R(E)-T C(E)
$$

donde TR son los ingresos totales y TC, los costos totales, ambos en función del esfuerzo de pesca.

El ingreso total será calculado usando la fórmula:

$$
T R=p . H
$$

donde $p$ denota el precio promedio por tonelada de pescado.

La relación entre costo y esfuerzo se asume lineal, entonces el esfuerzo total del esfuerzo de pesca será definido como:

$$
T C=c . E
$$

donde $c$ denota el costo unitario del esfuerzo que incluye el costo de oportunidaa del trabajo y del capital y $E$ la unidad de esfuerzo.

El rendimiento máximo económico $(M E Y)^{7}$ se obtiene en el nivel de esfuerzo que maximiza el beneficio al tomar la derivada de la ecuación (14) e igualando a cero la renta económica marginal: $\pi^{\prime}(E)=0$, lo que determina que: $d T R / d E=d T C / d E$; lo cual significa que el máximo beneficio se alcanza cuando el ingreso marginal $M R$ es igual al costo marginal $M C$. (Ver figura 5).

Para desarrollar las derivadas que expresan el ingreso marginal y el costo marginal, previamente hay que igualar: $T R=T C$ por sus ecuaciones equivalentes: (15) y (16), respectivamente, pero en el caso de (15), se sustituye la captura $H$ por su equivalente (8), quedando la ecuación como sigue:

$$
p\left(a E-b E^{2}\right)=c . E
$$

tomando la derivada con respecto a $E$ en ambos lados de la ecuación (17), se obtiene el esfuerzo en el rendimiento máximo económico:

$$
E_{M E Y}=\frac{\left(a-\frac{c}{p}\right)}{2 b}
$$

La biomasa en el rendimiento máximo económico $\left(X_{M E Y}\right)$, se obtiene reemplazando (18) en la ecuación (12):

\footnotetext{
${ }^{7}$ Maximum Economic Yield (MEY)
} 
Figura 5. Curvas de captura, ingresos y costos, según modelo Gor Schaefer

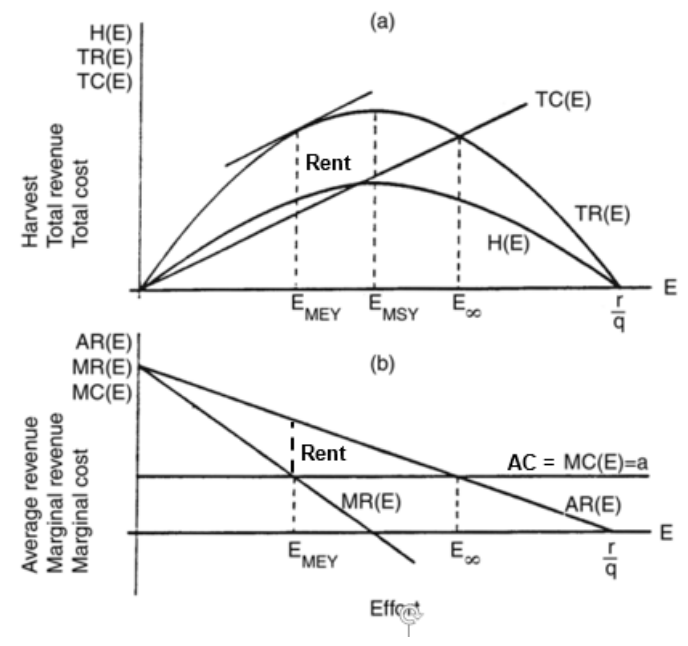

NOTA: El Panel (a) muestra las curvas de ingreso total (TR), costo total (TC) y captura sostenible $(\mathrm{H})$. El Panel (b), grafica las curvas de ingreso marginal (MR), ingreso promedio (AR), costo marginal (MC), costo promedio (AC)

Fuente: Flaaten (2014)

$$
X_{M E Y}=\frac{K}{2}+\frac{c}{2 p q}
$$

O, lo que es lo mismo (Figura 6) :

$$
X_{M E Y}=X_{M S Y}+\frac{1}{2} X_{\infty}
$$

\footnotetext{
${ }^{8} X_{\infty}$ corresponde al nivel de biomasa en un régimen de libre acceso, cuando el ingreso total es igual al costo total, es decir, cuando no hay renta económica.
} 
La captura en el rendimiento máximo económico $X_{M E Y}$ se obtiene reemplazando (18) en (8):

$$
H_{M E Y}=\frac{a^{2} p^{2}-c^{2}}{4 b p^{2}}
$$

El modelo bioeconómico estático de Gordon-Schaefer, aporta cuatro aspectos importantes para la gestión pesquera desde el punto de vista de la eficiencia pesquera o rendimiento máximo económico: i) determina la renta económica que puede aportar un determinado recurso; ii) demuestra que la renta económica es más alta no en el esfuerzo pesquero de rendimiento máximo sostenible, $E_{M S Y}$, sino en un nivel menor de esfuerzo pesquero, donde el ingreso marginal es igual al costo marginal (Figura 5, panel b), es decir, cuando se alcanza el máximo beneficio neto; iii) permite determinar el nivel de biomasa en el rendimiento máximo económico, $X_{M E Y}$ y iv) determina el nivel de captura en el rendimiento máximo económico.

\section{c) Modelo bioeconómico dinámico de Clark-Munro}

El modelo bioeconómico dinámico de Clark y Munro (1975), es una extensión del modelo estático de Gordon-Schaefer (Gordon, 1954 y Schaefer, 1957). Clark y Munro (1975), emplean un enfoque de la teoría moderna del capital y técnicas de la teoría del control óptimo aplicada a la economía pesquera. El fundamento de su enfoque está en ver a la población de peces o biomasa como un stock de capital, la cual -al igual que el capital hecho por el hombre o capital "convencional"- es capaz de generar un flujo de consumo (captura) sostenible a través del tiempo. Y, tal como ocurre con el capital "convencional", la decisión de consumo de hoy -por su

Figura 6. Curvas de ingreso total, costo total y renta del recurso, según modelo Gordon-Schaefer

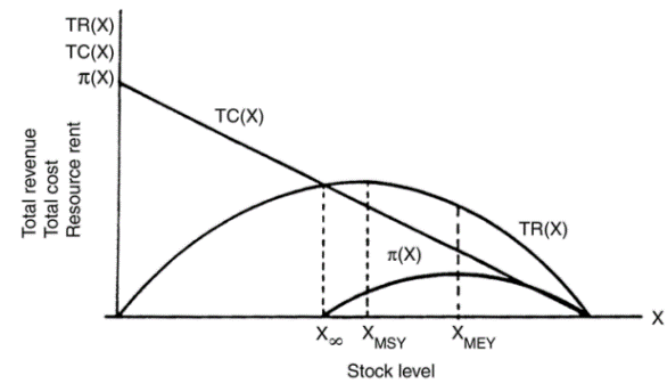

impacto en el nivel de stock (biomasa)- tendrá implicancias en las opciones futuras de consumo

NOTA: Ingreso Total (TR), Costo Total (TC) y Renta del Recurso

$[\pi(X)]$ como funciones de la biomasa.

Fuente: Flaaten, O. (2014)

(captura). Así, el problema de gestión del recurso, se convierte en uno de selección de un flujo de consumo (captura) óptimo, a través del tiempo, el cual a la vez, implica seleccionar un nivel de biomasa óptima como función del tiempo.

Tomando como base la ecuación de Schaefer (1), Clark y Munro (1975) asumen que la captura $h(t)$, es igual a la tasa de consumo y $d x / d t$, es la tasa de inversión (positiva o negativa) en el stock (biomasa), la cual corresponde a la diferencia entre el crecimiento natural del stock y la captura. 
En el marco de la teoría del control óptimo, el modelo de Clark y Munro (1975), considera la ecuación (1) como ecuación de estado. Y, dentro de dicha ecuación, $h(t)$, se convierte en variable de control. Sólo queda, entonces, definir la función objetivo, dado que estos tres elementos constituyen la base de la teoría del control óptimo, una extensión del cálculo de variaciones estándar que hizo posible el análisis de temas económicos dinámicos de una manera más completa. La teoría del control fue aplicada al análisis del crecimiento económico, inversión de capital, gestión de recursos naturales y otros temas que incluían la evaluación de los ingresos a través del tiempo (Flaaten, 2014).

El objetivo es maximizar el valor presente (PV) de la renta del recurso derivada de la captura. Dado los supuestos de precio constante y costos lineales en la captura, la función objetivo puede expresarse como:

$$
P V_{\pi}=\int_{0}^{\infty} e^{-\delta t} \cdot \pi(t) d t
$$

donde: $e^{-\delta t}$ es el factor de descuento en tiempo instantáneo o continuo y $\pi(t)$ la renta del recurso o beneficio de la pesquería, expresada como sigue:

$$
\pi(t)=p h(t)-c E(t)
$$

despejando $E$ de la ecuación (3) y reemplazando en (23), se tiene:

$$
\pi(t)=p h(t)-c \frac{h(t)}{\mathrm{qX}(\mathrm{t})}
$$

factorizando $h(t):{ }^{9}$

$$
\pi(t)=[p-c(X)] h(t)
$$

reemplazando la ecuación (23) en la ecuación (22), se tiene:

$$
P V_{\pi}=\int_{0}^{\infty} e^{-\delta t}[p-c(X)] h(t) d t
$$

Dado que la función objetivo es lineal en la variable de control $h(t)$, entonces se está frente a un problema de control óptimo lineal. El problema es determinar el control óptimo $h(t)=h^{*}(t), \quad t$ $\geq 0$, y la correspondiente población óptima, $x(t)=x^{*}(t), t \geq 0$, sujeto a la ecuación de estado (1) y a las restricciones de control: $\quad 0 \leq h(t) \leq h$ máx; tal que, la función objetivo (26) asume el máximo valor. El problema se resuelve por la vía del Principio del Máximo de Pontryagin, que aplica ecuaciones Hamiltonianas.

En consecuencia, según Clark y Munro (1975), la Hamiltoniana del problema está dada por:

\footnotetext{
${ }^{9} c(X)=c / q X(t)$, se convierte en costo unitario de la captura. Esto demuestra que el costo unitario de la captura decrece con un incremento del tamaño del stock, Un stock grande genera ahorro en costos.
} 


$$
H=e^{-\delta t}[\{p-c(x)\} h(t)+\psi(t)\{F(x)-h(t)\}]
$$

donde $\psi(t)$, es la variable adjunta o variable de coestado en valor corriente.

Multiplicando y factorizando $h(t)$, se tiene la ecuación:

$$
\begin{gathered}
H=e^{-\delta t}[p-c(x)-\psi(t)] h(t)+e^{-\delta t} \psi(t) F(x) \\
H=\sigma(t) h(t)+e^{-\delta t} \psi(t) F(x)
\end{gathered}
$$

donde, $\sigma(t)$, se denomina función de cambio y sustituye en la ecuación previa a:

$$
\sigma(t)=e^{-\delta t}[p-c(x)-\psi(t)]
$$

Para resolver el problema de control óptimo, primero, se determina la llamada solución singular $x^{*}$, tomando la derivada de la ecuación (28) y luego cuando $\sigma(t) \equiv 0$, se obtiene la siguiente ecuación:

$$
(1 / \delta)\left[\left(d / d x^{*}\right)\left\{\left(p-c\left(x^{*}\right)\right) F\left(x^{*}\right)\right\}\right]=p-c\left(x^{*}\right)
$$

Esta ecuación no involucra el tiempo t, explícitamente. Por tanto, la solución $X^{*}$ es constante y constituye una solución en estado de equilibrio.

Según Clark y Munro (1975), la ecuación (30), puede interpretarse sin dificultad. El lado izquierdo, es el valor presente de la renta marginal sostenible del recurso, $\left(d / d x^{*}\right)\{(p$ $\left.\left.c\left(x^{*}\right)\right) F\left(x^{*}\right)\right\}$, proporcionado por el incremento marginal del stock. El lado derecho es la renta marginal del recurso, disfrutada a partir de la captura actual.

Por una parte, el lado izquierdo de la ecuación (30) puede interpretarse como una expresión del costo marginal del usuario, el cual muestra el costo presente de la captura del incremento marginal del stock, un costo que tiene que ser ponderado contra la ganancia marginal de la captura actual. Y, por otra parte, los lados, izquierdo y derecho de la ecuación, pueden verse como el precio de demanda imputado y el precio de oferta, respectivamente, del "activo" de capital en el tiempo $t$.

Una forma más transparente de (30) se obtiene llevando a cabo la diferenciación en el lado izquierdo y luego multiplicando por $\delta /\left(p-c\left(x^{*}\right)\right)$ :

$$
F^{\prime}\left(x^{*}\right)-\left[c^{\prime}\left(x^{*}\right) F\left(x^{*}\right) /\left(p-c\left(x^{*}\right)\right)\right]=\delta
$$

La ecuación [31] indica que el stock óptimo $x^{*}$ es aquel cuya tasa de interés es igual a la tasa social de descuento $\delta$. Es también una ecuación implícita para la población $x *$. Se asume que dicha ecuación tiene una solución única $x=x^{*}$, denominada nivel de población óptima (equilibrio).

Asimismo, la ecuación [31] "es la ecuación de la teoría del capital, que responde a la cuestión del nivel de stock óptimo del capital natural, $x^{*}, \mathrm{y}$, como tal, es una versión de la Regla de Oro Modificada de la Acumulación de Capital de la teoría básica del capital (Clark y Munro, 1975). Dice simplemente, invierta en $x$, hasta el punto en que la "tasa de interés propia" (o tasa interna de retorno) de la inversión marginal en $x$ sea igual a la tasa social de descuento" (Clark y Munro, 2017).

Según Clark (1990), la solución de la ecuación (31), conduce a una función cuadrática donde el nivel de stock óptimo $x^{*}$, es una raíz cuadrada positiva y depende de los parámetros $c, p, q, r$, $\begin{array}{llll}k & y & \delta & \text {, conforme a la siguiente relación: }\end{array}$ 
Figura 7. Biomasa y captura óptimas, según modelo Clark-Munro

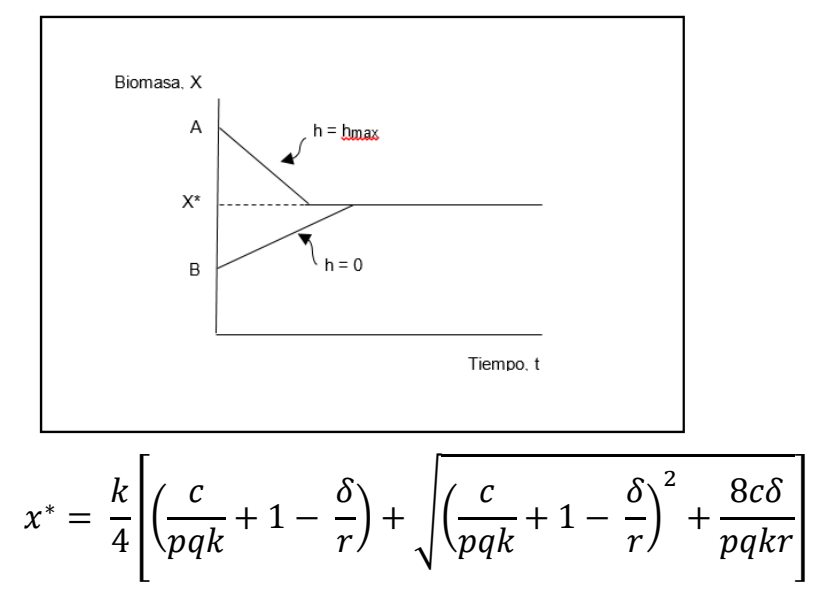

El rendimiento óptimo sostenible $\left(O S Y^{10}\right)$ y el nivel de esfuerzo óptimo sostenible $\left(E_{O S Y}\right)^{11}$, para el precio del tiempo o tasa de descuento especificada $\delta$, se obtiene con las siguientes expresiones, respectivamente:

$$
\begin{gathered}
O S Y=r \cdot x^{*}\left(1-\frac{x^{*}}{K}\right) \\
E_{O S Y}=\frac{O S Y}{q \cdot x^{*}} \\
h^{*}(t) \begin{cases}h_{\text {max }} & \text { If } x>x^{*} \\
F\left(x^{*}\right), & \text { If } x=x^{*} \\
0, & \text { If } x<x^{*}\end{cases}
\end{gathered}
$$

En base a la determinación de la captura óptima y el esfuerzo óptimo, y el precio de venta de la captura y el costo del esfuerzo pesquero, se determina la renta del recurso óptima y se estima los derechos de pesca del recurso anchoveta para un nivel óptimo de biomasa.

Acompaña al modelo de Clark-Munro la ecuación de la teoría de la inversión (35), que prescribe la tasa óptima de inversión/desinversión en $x$. Indica que, si $x(t)<x^{*}$, la tasa óptima de inversión positiva en el recurso, es la tasa máxima, si $x(t)>x^{*}$, la tasa óptima de desinversión, es la tasa máxima.

La Figura 7 muestra la representación de la ecuación (35):

El modelo de optimización de Clark-Munro, sugiere invertir en el recurso en caso que la biomasa óptima estimada resulte superior a la biomasa actual, a fin de alcanzar, tan rápido como sea posible, el nivel poblacional óptimo. En este caso, invertir en el recurso, de acuerdo a Scott

Fuente: Clark (1990)

\footnotetext{
${ }^{10}$ Optimal Sustainable Yield (OSY). En algún momento se usará la notación $H^{*}$ (captura óptima)

${ }^{11}$ En algún momento se usará la notación $E^{*}$ (Esfuerzo óptimo)
} 
(1955), significa cerrar la zona de pesca completamente y suspender temporalmente la actividad pesquera $(h=0)$, a fin de permitir la reconstrucción del recurso. Al respecto, Clark y Munro (2017) señalan que si bien el programa de inversión prescrito por la ecuación (35), en el caso de los recursos pesqueros que deben ser reconstruidos, es draconiano, debe declararse y mantenerse hasta que se alcance la meta $X^{*}$, aun si esto toma décadas; y añaden que si se hacen concesiones a la política draconiana, tendrían que hacerse en términos políticos y sociales y no en términos económicos. Pero, también agregan que el programa draconiano de inversión en el recurso, es válido en términos económicos sólo bajo condiciones muy especiales, como por ejemplo, que tanto el capital producido como el capital humano sean perfectamente maleables, es decir, que ambos capitales puedan ser movidos con facilidad y sin costo significativo dentro 0 fuera de la pesquería. Si el capital producido es menos que perfectamente maleable, no es óptimo, en términos reales, declarar una moratoria completa de la captura para los recursos pesqueros a ser reconstruidos, excepto en casos de extrema sobrexplotación y sólo por un período limitado (Clark, et al., 1979).

\section{METODOLOGÍA}

El presente estudio se enmarca dentro del enfoque cuantitativo de la investigación científica y de diseño no experimental y longitudinal de tendencia. Se fundamenta en la estimación de la renta del recurso, a través del modelo bioeconómico de optimización dinámica en tiempo continuo, formulado por Clark y Munro (1975).

\section{Área de estudio}

Se estableció como área de estudio la zona norte-centro del litoral, comprendida entre los $03^{\circ} 30^{\prime}-16^{\circ} \mathrm{S}$. Se evaluó la captura y el esfuerzo pesquero de la flota anchovetera de mayor escala (embarcaciones implementadas con red de cerco, mayores de 32,6 $\mathrm{m} 3$ de capacidad de bodega) durante el período 2004-2017.

\section{Selección y estandarización del esfuerzo pesquero}

La selección de la medida del esfuerzo pesquero es clave en la investigación. Es el primer paso en el tratamiento de los datos de captura y esfuerzo, el cual va acompañado de un proceso de estandarización del esfuerzo, pues los tamaños de las embarcaciones anchoveteras son muy disímiles y pueden influir en el cálculo de la CPUE del total de embarcaciones. La captura y el esfuerzo pesquero estandarizado, permiten estimar los coeficientes de captura $a$ y $b$ de la ecuación (9), mediante un modelo de regresión lineal simple, que considera la relación de dependencia entre la variable dependiente "captura por unidad de esfuerzo" (CPUE) y la variable independiente "esfuerzo pesquero".

La curva de regresión lineal con pendiente negativa, confirma el resultado de una adecuada selección del esfuerzo pesquero. Gordon (1954) señala una relación uniformemente lineal, entre la CPUE y el esfuerzo (Figura 4).

A partir de los datos del "Programa de Vigilancia y Control de la Pesca y Desembarque en el Ámbito Marítimo", se selecciona el esfuerzo pesquero en términos de $\mathrm{m}^{3}$-desembarque, que cumple con la condición señalada en el párrafo anterior. El esfuerzo pesquero, en los términos señalados, se obtiene multiplicando la capacidad de bodega en metros cúbicos por el número de desembarques de cada embarcación por mes. Para este efecto, se agrupa las embarcaciones anchoveteras según su capacidad de bodega en metros cúbicos, de acuerdo a la siguiente estratificación: i) entre $32,6 \mathrm{~m}^{3}-130 \mathrm{~m}^{3}$; ii) entre $131 \mathrm{~m}^{3}$ y $450 \mathrm{~m}^{3}$; $\mathrm{y}$, iii) mayores de $450 \mathrm{~m}^{3}$. Se utilizó como criterio de agrupación la similitud de las cantidades totales desembarcadas por los grupos i) y iii); cada uno desembarca al año alrededor del $20 \%$ del total del recurso anchoveta; y, el grupo ii), el 60\%, según el período de extracción pesquera estudiado (2004-2017).

El proceso de estandarización del esfuerzo pesquero, se realizó mediante el procedimiento de Beverton y Holt (1993), según el cual, se selecciona, en primer término, un grupo como estándar y se calcula su correspondiente CPUE; luego, se asigna a cada grupo restante un factor derivado del ratio entre la CPUE de un grupo y la CPUE del grupo tomado como estándar. EI esfuerzo estandarizado de cada grupo, se obtiene al multiplicar el esfuerzo simple por su factor. 
Y, el esfuerzo total anual estandarizado será la suma de los esfuerzos estandarizados de cada uno de los grupos establecidos. Se entiende que las capturas se han realizado en el mismo caladero y con la misma densidad de peces.

\section{Obtención de la captura por unidad de esfuerzo (CPUE) estandarizada}

La captura por unidad de esfuerzo (CPUE) estandarizada anual, se obtiene dividiendo la captura anual entre el esfuerzo pesquero anual estandarizado.

\section{Estimación de los coeficientes de captura por unidad de esfuerzo $a$ y $b$}

Los valores anuales de la CPUE estandarizada y del esfuerzo pesquero estandarizado, permiten estimar los coeficientes de captura por unidad de esfuerzo $a$ y $b$ de la ecuación (9), a través de un modelo de análisis de regresión lineal simple.

\section{Estimación de parámetros biológico y tecnológico}

A partir de los coeficientes de captura por unidad de esfuerzo $a$ y $b$, obtenidos mediante análisis de regresión lineal simple de los datos anuales de la CPUE estandarizada y del esfuerzo estandarizado, se estima los parámetros biológico y tecnológico: la capacidad de carga del hábitat $(K)$ y coeficiente de capturabilidad $(q)$, respectivamente. Ambos parámetros se asumen fijos (Flaaten, 2014) y son clave para la aplicación del modelo bioeconómico dinámico de Clark y Munro (1975).

\section{Cálculo del MSY, MEY y OSY}

El cálculo del Rendimiento Máximo Sostenible (MSY) y del Rendimiento Máximo Económico (MEY) y sus correspondientes niveles de esfuerzo pesquero y biomasa, se efectúa con fines referenciales, a partir del modelo Verhulst-Schaefer y del modelo Gordon-Schaefer, respectivamente.

El Rendimiento Óptimo Sostenible (OSY) y su correspondiente nivel de esfuerzo óptimo $\left(E^{\star}\right)$ y biomasa óptima $\left(X^{\star}\right)$, se determina aplicando el modelo dinámico en tiempo continuo de Clark y Munro (1975), a partir del cual se calcula la renta del recurso.

El eje central del modelo Clark-Munro, gira en torno al cálculo de la biomasa óptima $\left(X^{\star}\right)$, a partir de la cual, se determina la captura óptima $\left(H^{\star}\right)$ y el esfuerzo óptimo $\left(E^{\star}\right)$. El nivel de biomasa óptima se convierte así en el objetivo a ser alcanzado por el administrador de una pesquería, manejando la captura como variable de control.

"Esencialmente, el nivel de stock óptimo $\left(X^{\star}\right)$ se determina por parámetros biológicos $(r, K)$, tecnológicos $(q)$ y económicos ( $p, c, \delta$ )" (Bjørndal, y Bezabih, 2010).

\section{Cálculo de la renta del recurso óptima}

La renta del recurso óptima $\left(\pi^{*}\right)$, se calcula aplicando la ecuación (23), considerando la captura óptima $\left(H^{\star}\right)$ y el esfuerzo óptimo $\left(E^{\star}\right)$.

\section{Cálculo del derecho de pesca}

El derecho de pesca (FF) se calcula mediante la ecuación aplicada por Viet Thanh (2006) en su análisis bioeconómico de la pesquería del langostino en el Golfo de Tonkin, Vietnam, la cual se ilustra en la Figura 10.

En términos unitarios, el derecho de pesca estaría dado por la diferencia entre el precio de la captura y el costo del esfuerzo en el nivel del rendimiento óptimo sostenible (OSY).

\section{Diseño muestral}

El presente trabajo no considera una muestra en el proceso de investigación, sino el total de capturas del recurso anchoveta con destino al consumo humano indirecto realizadas en el área de estudio, esto es, en la Zona Norte-Centro del litoral comprendida entre los $03^{\circ} 30^{\prime}-16^{\circ} \mathrm{S}$, durante el período 2004-2017, así como el esfuerzo pesquero aplicado a dichas capturas por la 
flota anchovetera de mayor escala (embarcaciones implementadas con red de cerco, mayores de 32,6 m3 de capacidad de bodega).

\section{Técnicas de recolección de datos}

Los datos de captura y de esfuerzo pesquero, se han obtenido de los registros del "Programa de Vigilancia y Control de la Pesca y Desembarque en el Ámbito Marítimo", que se viene ejecutando desde el año 2004 a través de empresas privadas de inspección por encargo de la autoridad pesquera, conforme al Decreto Supremo № 027-2003-PRODUCE. La información sobre montos anuales de derechos de pesca del recurso anchoveta fue proporcionada por el Ministerio de la Producción.

Los precios de mercado del recurso anchoveta, se obtuvieron de los reportes bianuales sobre mercados globales de alimentos de FAO (2018) y FAO (2015) y, asimismo, se tomaron en consideración los estudios académicos realizados por De La Puente et al. (2011), Paredes (2012) y Galarza y Collado (2013). Los costos del esfuerzo pesquero en términos de m3-desembarque, se deducen a partir de los costos de captura (US\$/TM) consignados en los estudios académicos de De La Puente et al. (2011), Paredes (2012) y Galarza y Collado (2013), así como de información obtenida de una empresa pesquera del sector privado.

\section{Técnicas estadísticas para el procesamiento de la información}

Se utilizó la técnica de regresión lineal simple para obtener los coeficientes de captura por unidad de esfuerzo $a$ y $b$ del modelo bioeconómico dinámico a aplicarse, a partir de los datos históricos de captura y de esfuerzo pesquero del recurso anchoveta de la zona norte-centro, correspondiente al período 2004-2017.

\section{ANÁLISIS Y DISCUSIÓN DE RESULTADOS}

\section{Estimación del esfuerzo pesquero estandarizado y de la CPUE estandarizada}

La Tabla 3, muestra los datos históricos anuales de captura de anchoveta del período 20042017 de la zona norte-centro, por grupos de embarcaciones pesqueras anchoveteras de mayor escala, así como la estimación del esfuerzo pesquero estandarizado y de su correspondiente CPUE estandarizada, para el total de la flota anchovetera. La medida de selección del esfuerzo pesquero resultó compatible sólo con el régimen de pesca de acceso abierto regulado, 20042008 (prereforma), por tanto, el modelo sólo se aplicará a este régimen.

Tabla 3. Captura, esfuerzo pesquero estandarizado y CPUE estandarizada

\begin{tabular}{|c|c|c|c|c|c|c|c|c|c|}
\hline \multirow[b]{2}{*}{ Año } & \multicolumn{2}{|c|}{$\begin{array}{c}\text { Grupo } 1 \\
\text { Tamaño E/P [32,6-130 m3] }\end{array}$} & \multicolumn{2}{|c|}{$\begin{array}{c}\text { Grupo } 2 \\
\text { Tamaño E/P [131 - 450 m3] }\end{array}$} & \multicolumn{2}{|c|}{$\begin{array}{c}\text { Grupo } 3 \\
\text { Tamaño E/P }[>450 \mathrm{~m} 3 \\
\end{array}$} & \multicolumn{3}{|c|}{$\begin{array}{c}\text { Total } \\
\text { Flota anchovetera }\end{array}$} \\
\hline & $\begin{array}{l}\text { Captura } \\
\text { ( TM) }\end{array}$ & $\begin{array}{c}\text { Esfuerzo } \\
\text { estandarizado } \\
\text { (m3-desembarque) }\end{array}$ & $\begin{array}{l}\text { Captura } \\
\text { (TM) }\end{array}$ & $\begin{array}{c}\text { Esfuerzo } \\
\text { estándar } \\
\text { (m3-desembarque) }\end{array}$ & $\begin{array}{l}\text { Captura } \\
\text { (TM) }\end{array}$ & $\begin{array}{c}\text { Esfuerzo } \\
\text { estandarizado } \\
\text { (m3-desembarque) }\end{array}$ & $\begin{array}{l}\text { Captura } \\
\text { (TM) }\end{array}$ & $\begin{array}{c}\text { Esfuerzo } \\
\text { estandarizado } \\
\text { (m3-desembarque) }\end{array}$ & $\begin{array}{c}\text { CPUE } \\
\text { estandarizado }\end{array}$ \\
\hline 2004 & $1,356,956$ & $3,050,780$ & $5,109,246$ & $11,281,101$ & $1,136,941$ & $2,502,952$ & $7,603,143$ & $16,834,833$ & 0.452 \\
\hline 2005 & $1,430,028$ & $2,750,638$ & $4,986,740$ & $9,605,759$ & $1,193,272$ & $2,276,552$ & $7,610,039$ & $14,632,949$ & 0.520 \\
\hline 2006 & $1,042,131$ & $1,598,141$ & $3,156,182$ & $5,014,063$ & 788,130 & $1,246,609$ & $4,986,443$ & $7,858,812$ & 0.635 \\
\hline 2007 & $1,302,145$ & $2,161,988$ & $3,042,927$ & $5,003,286$ & 774,293 & $1,278,133$ & $5,119,365$ & $8,443,407$ & 0.606 \\
\hline 2008 & $1,174,872$ & $1,836,226$ & $3,276,369$ & $5,270,454$ & 837,945 & $1,358,481$ & $5,289,186$ & $8,465,160$ & 0.625 \\
\hline 2009 & $1,191,069$ & $2,187,806$ & $3,168,517$ & $5,705,949$ & $1,089,172$ & $1,941,447$ & $5,398,758$ & $9,835,202$ & 0.549 \\
\hline 2010 & 706,117 & $1,280,459$ & $1,838,562$ & $3,377,417$ & 721,175 & $1,316,277$ & $3,265,855$ & $5,974,153$ & 0.547 \\
\hline 2011 & $1,292,844$ & $2,083,599$ & $3,385,448$ & $5,467,179$ & $1,460,755$ & $3,884,033$ & $6,139,047$ & $11,434,811$ & 0.537 \\
\hline 2012 & 666,929 & $1,638,070$ & $1,830,495$ & $4,544,016$ & 868,595 & $2,092,449$ & $3,366,019$ & $8,274,535$ & 0.407 \\
\hline 2013 & 863,297 & $1,605,893$ & $2,366,518$ & $4,310,176$ & $1, \propto 22, \infty 27$ & $1,868,690$ & $4,251,841$ & $7,784,760$ & 0.546 \\
\hline 2014 & 334,137 & $1,006,363$ & 927,844 & $2,634,434$ & 431,429 & $1,273,310$ & $1,693,410$ & $4,914,106$ & 0.345 \\
\hline 2015 & 706,281 & $1,666,926$ & $2,013,631$ & $4,657,326$ & 894,721 & $2,148,860$ & $3,614,633$ & $8,473,112$ & 0.427 \\
\hline 2016 & 565,186 & $1,831,214$ & $1,623,663$ & $4,839,682$ & 687,908 & $2,040,114$ & $2,876,757$ & $8,711,010$ & 0.330 \\
\hline 2017 & 705,176 & $1,914,094$ & $1,627,251$ & $4,290,936$ & 727,174 & $1,942,425$ & $3,059,600$ & $8,147,455$ & 0.376 \\
\hline
\end{tabular}

Estimación de los coeficientes de captura por unidad de esfuerzo $a$ y $b$ 
Los resultados de la estimación de los coeficientes de captura por unidad de esfuerzo $a$ y $b$ de la ecuación (9), se muestran en la Tabla 4. Se aplicó la técnica de regresión lineal simple. Los resultados indican una relación negativa esperada entre los valores estandarizados de la CPUE y el esfuerzo pesquero, para el período 2004-2008, tal como se muestra en la Figura 8 . Este resultado permite verificar una adecuada selección de la medida de esfuerzo pesquero. El valor del coeficiente $(b)$, igual a $-2 \mathrm{E}-08$, expresa la pendiente negativa y el valor del coeficiente $(a)$, 0,7776 , corresponde a la intersección con el eje de las ordenadas. El valor $\mathrm{R}^{2}$ de 0,9756 indica que el $97 \%$ de las variaciones de la CPUE se explican por la variable explicativa del esfuerzo pesquero estandarizado.

Figura 8. Estimación de los coeficientes de captura $a$ y $b$

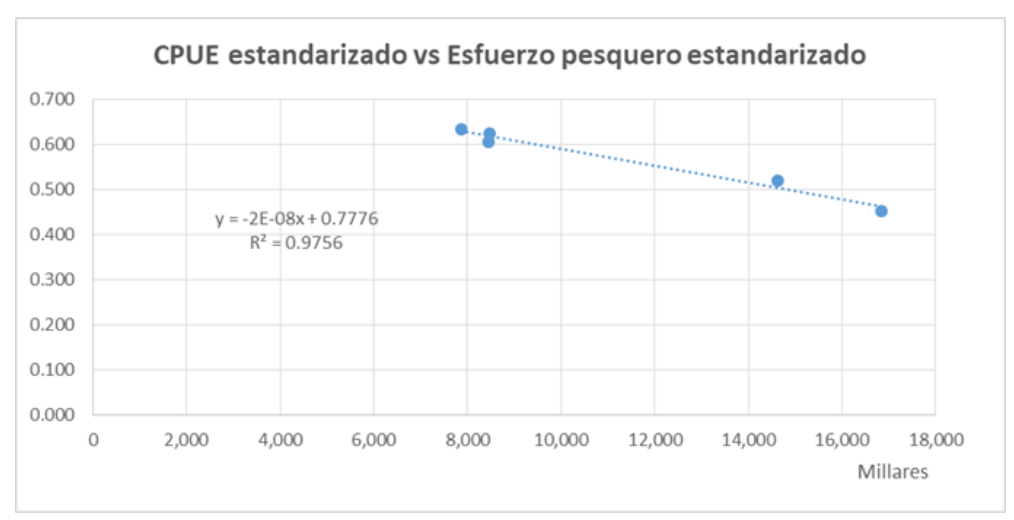

Tabla 4. Coeficientes estimados de captura por unidad de esfuerzo $a$ y $b$

\begin{tabular}{c|c|c|c}
\hline Modelo & $a$ & $b$ & $\mathbf{R}^{2}$ \\
\hline \hline Verhulst-Schaefer & 0,7776 & $-2 \mathrm{E}-08$ & 0,9756 \\
\hline
\end{tabular}

\section{Estimación de los parámetros biológicos y tecnológicos $K$ y $q$}

La estimación de los parámetros biológicos y tecnológicos, denominados capacidad de carga $(K)$ y coeficiente de capturabilidad $(q)$, respectivamente, se realizó aplicando los coeficientes de captura por unidad de esfuerzo $(a)$ y $(b)$ obtenidos por regresión lineal (Tabla 4) y la tasa intrínseca de crecimiento ( $r$ ) reportada por Oliveros (2009), conforme se muestra en la Tabla 10. Los parámetros $(r)$ y $(K)$ se asumen fijos (Flaaten, 2014) en los modelos bioeconómicos y biológicos.

Tabla 5. Estimación de los parámetros biológicos y tecnológicos $K$ y $q$

\begin{tabular}{|c|c|c|}
\hline$a=q K$ & $K=\frac{a}{q}$ & $K=31.657 .684$ toneladas \\
\hline$b=\frac{q^{2} K}{r}$ & $b=\frac{q^{2}\left(\frac{a}{q}\right)}{r}$ & $q=2,45628 E-08 \quad$ año $\left(m^{3}\right)^{-1}$-desemb ${ }^{-1}$ \\
\hline
\end{tabular}

$r=0,955$ año $^{-1}$, Oliveros (2009)

Cabe anotar que, el valor estimado de la capacidad de carga del hábitat, $K=31.657 .684$ toneladas, a partir de los datos empíricos de captura y esfuerzo pesquero, correspondientes al 
período de pesca 2004-2008, es bastante aproximado al valor $K=33.000 .000$ toneladas, señalado por Oliveros (2009).

Asimismo, el valor estimado del coeficiente de capturabilidad, $q=2,45628 \mathrm{E}-08$ año $(\mathrm{m} 3)^{-1}$ desembarque ${ }^{-1}$, se encuentra dentro del rango de los resultados obtenidos por Csirke (1989a) en su estudio sobre fluctuaciones estacionales del coeficiente de capturabilidad de la pesquería de la anchoveta peruana, para el período de estudio 1960-1971.

\section{Cálculo del MSY, MEY, OSY y sus correspondientes niveles de esfuerzo y biomasa}

Para el cálculo del Rendimiento Máximo Sostenible (MSY), el Rendimiento Máximo Económico (MEY) y el Rendimiento Óptimo Sostenible (OSY), así como de sus correspondientes niveles de esfuerzo pesquero y de biomasa, se consideró, según el caso, los coeficientes de captura por unidad de esfuerzo $a$ y $b$, así como los parámetros biológicos $r$ y $K$, y el parámetro tecnológico $q$, así como los parámetros económicos: precio $(p)$ del recurso en el desembarque, expresado en dólares, costo unitario $(c)$ del esfuerzo pesquero, expresado en dólares $/ \mathrm{m}^{3}$ desembarque, tasa social de descuento y el promedio ponderado del costo de capital (WACC ${ }^{12}$ por sus siglas en inglés); todos ellos se muestran en la Tabla 6.

Cabe indicar que, debido a la falta de registros oficiales sobre costos de captura del recurso anchoveta, se optó por considerar como costo unitario promedio de captura $(C)$, para el período 2004-2008, el costo unitario de captura promedio ponderado de US\$ 85/t, resultante del costo mencionado por Galarza y Collado (2013) para el año 2006 de US\$ 76,31 por tonelada, el cual incluye el costo de oportunidad del capital (WACC) y el costo unitario reportado por De la Puente, et al. (2011) para la primera temporada de pesca del año 2009, bajo el supuesto que dicho costo aun respondería al patrón característico del régimen prereforma del año 2008. A dicho costo unitario se le sumó el corresponde costo de oportunidad del capital (13\%), arrojando el valor de US\$ 93,27/t. El costo unitario de captura $(C)$ permite estimar el costo unitario del esfuerzo (c) estableciendo la igualdad entre el costo total de captura y el costo total del esfuerzo.

Tabla 6. Coeficientes de captura y parámetros biológicos y económicos 2004-2008

\begin{tabular}{c|l|c|c}
\hline Símbolo & \multicolumn{1}{|c|}{ Definición } & Unidades & Valor \\
& & & \\
\hline \hline$a$ & Coeficiente de captura (intersección) & constante & 0,7776 \\
\hline$b$ & Coeficiente de captura (pendiente) & constante & $-2 \mathrm{E}-08$ \\
\hline$r$ & Tasa intrínseca de crecimiento & año ${ }^{-1}$ & 0,955 \\
\hline$q$ & Coeficiente de capturabilidad & año $\left(\mathrm{m}^{3}\right)^{-1}-$ desembarque ${ }^{-1}$ & $2,45628 \mathrm{E}-08$ \\
\hline$K$ & Capacidad de carga del hábitat & toneladas & 31.657 .684 \\
\hline$p$ & Precio promedio del recurso & US $/ \mathrm{t}$ & 128 \\
\hline$C$ & Costo unitario promedio de captura & US $/ \mathrm{t}$ & 85 \\
\hline$c$ & Costo unitario promedio del esfuerzo & US $\$ / \mathrm{m}^{3}-$ desembarque & 46,26 \\
\hline$\delta$ & Tasa social de descuento promedio & $\%$ & 10,596 \\
\hline WACC & Promedio ponderado del costo de capital & $\%$ & 13 \\
\hline
\end{tabular}

La tasa social de descuento $(\delta)$ corresponde a la tasa aplicada en proyectos de inversión pública, calculada con la rentabilidad real ponderada del capital, utilizando todos los instrumentos de ahorro del mercado (Ministerio de Economía y Finanzas, 2012). Para los fines del presente trabajo, se calculó la tasa anual promedio del período 2004-2008.

Para el caso del WACC, se tomó como referencia la tasa aplicada por Galarza y Collado (2013). Y, en el caso del precio del recurso en el desembarque, se calculó el promedio ponderado del precio de pelágicos, excepto el atún, publicados por FAO (2015) y FAO (2018).

\section{Cálculo del Rendimiento Máximo Sostenible (MSY)}

\footnotetext{
${ }^{12}$ Weighted Average Cost of Capital (WACC)
} 
La Tabla 7 muestra los resultados del rendimiento máximo sostenible $\left(H_{M S Y}\right)$ del recurso anchoveta y sus correspondientes niveles de esfuerzo pesquero y biomasa de la zona nortecentro para el período 2004-2008, calculados conforme al modelo biológico de VerhulstSchaefer.

Tabla 7. Cálculo del Rendimiento Máximo Sostenible

\begin{tabular}{c|c|c|c}
\hline Modelo & $\begin{array}{c}\text { Captura }(\boldsymbol{t}) \\
H_{M S Y}=a^{2} / 4 b\end{array}$ & $\begin{array}{c}\text { Esfuerzo } \\
(\mathbf{m} 3 \text {-desembarque }) \\
E_{M S Y}=a / 2 b\end{array}$ & $\begin{array}{c}\text { Biomasa }(\boldsymbol{t}) \\
X_{M S Y}=K / 2\end{array}$ \\
\hline \hline Verhulst-Schaefer & 7.558 .272 & 19.440 .000 & 15.828 .842 \\
\hline
\end{tabular}

La aplicación del modelo Verhulst-Schaefer, determina que la captura $\left(H_{M S Y}\right)$ o rendimiento máximo sostenible, es de 7.558.272 toneladas y que ésta se genera a partir de una biomasa de 15.828.842 toneladas. Cabe indicar que, Csirke (1989) registró niveles mensuales estimados de biomasa promedio de anchoveta entre 13,2 y 17,7 millones de toneladas en la zona norte-centro del litoral, el año 1967, mientras que Oliveros (2010), reporta biomasas medias anuales estimadas en 18,5 millones de toneladas los años 1969-1970.

Analizando los datos históricos de captura de anchoveta en la zona norte-centro, que se muestran en la Tabla 3, se verifica que la captura $\left(H_{M S Y}\right)$, ocurrió los años 2004 y 2005, correspondientes al régimen de cuota total de captura, en los cuales se desembarcó 7.603 .143 y 7.603.039 toneladas, respectivamente. Durante el régimen de cuotas individuales de pesca (2009-2017), se verifica que no se alcanzó el nivel de rendimiento máximo sostenible, pues el más alto nivel de desembarque de anchoveta fue de 6.139.047 toneladas, obtenido el año 2011, siendo el desembarque promedio anual de dicho período de 3,74 millones de toneladas, mientras que el desembarque promedio anual del período 2004-2008, fue de 6,12 millones de toneladas de anchoveta.

Cabe señalar que, los años 2004 y 2005, en los que se alcanzó desembarques de anchoveta en el nivel de rendimiento máximo sostenible, las biomasas hidroacústicas anuales fueron de 12,7 y 12,5 millones de toneladas, respectivamente, según los niveles de biomasa por temporada de pesca estimados por Imarpe ${ }^{13}$; ambos volúmenes, algo más bajos que la biomasa calculada mediante el modelo Verhulst-Schaefer (Tabla 7).

Por otra parte, se verificó que la medida seleccionada de esfuerzo pesquero $\left(\mathrm{m}^{3}\right.$ desembarques), permite cumplir, para el período $2004-2008$, con el segundo requisito del modelo de Verhulst-Schaefer, referido a la formación de una curva cuadrática o parabólica, expresada en la ecuación (8) que relaciona los niveles de captura y esfuerzo. La Figura 9 muestra esta relación con un $R^{2}=0,9954$ y confirma el rendimiento máximo sostenible $\left(H_{M S Y}\right)$ y el esfuerzo $\left(E_{M S Y}\right)$, calculados mediante las ecuaciones del modelo Verhulst-Schaefer.

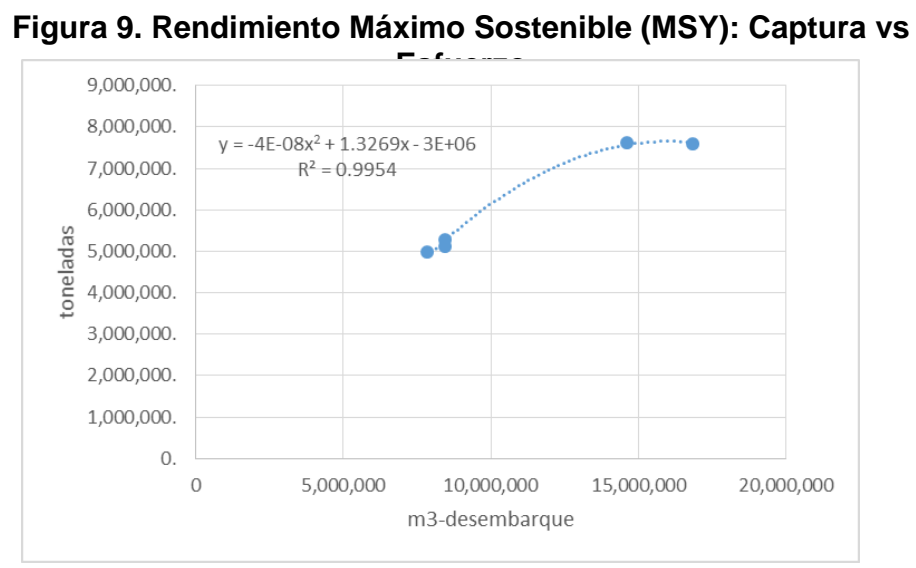

\footnotetext{
${ }^{13}$ Memorando N 048-2018-IMARPE/DGIRP de 22 de enero de 2018.
} 


\section{Cálculo del Rendimiento Máximo Económico (MEY)}

El rendimiento máximo económico $\left(H_{M E Y}\right)$ del recurso anchoveta y sus correspondientes niveles de esfuerzo pesquero y biomasa de la zona norte-centro para el período 2004-2008, calculados de acuerdo al modelo bioeconómico estático de Gordon-Schaefer, se muestran en la Tabla 8.

Según Clark (como se citó en Clarke et al., 1992), la idea detrás del MEY es que la pesquería se maneja como un bien de capital que maximiza el valor presente neto [de un período discreto].

Tabla 8. Cálculo del Rendimiento Máximo Económico

\begin{tabular}{c|c|c|c|c}
\hline Modelo & Captura ( $t)$ & $\begin{array}{c}\text { Esfuerzo } \\
\text { (m3-desembarque) }\end{array}$ & Biomasa ( $t)$ & $\begin{array}{c}\text { Renta } \\
\pi \\
(\text { USS })\end{array}$ \\
\hline \hline $\begin{array}{c}\text { Gordon- } \\
\text { Schaefer }\end{array}$ & 5.916 .558 & 10.379 .883 & 23.205 .959 & 275.086 .669 \\
\hline
\end{tabular}

Se puede advertir que, la captura $\left(H_{M E Y}\right)$ es $21,72 \%$ más baja que la captura $\left(H_{M S Y}\right) ; y$, el esfuerzo $\left(E_{M E Y}\right), 46,60 \%$ más bajo que el esfuerzo $\left(E_{M E Y}\right)$. Esto significa que, con menor esfuerzo es posible maximizar la renta del recurso, pero siempre que se consiga incrementar la biomasa $\left(X_{M E Y}\right)$ en un $46,60 \%$ con respecto a la biomasa $\left(X_{M S Y}\right)$. La renta del recurso $(\pi)$ en el nivel del máximo rendimiento económico, se calcula a partir de la ecuación (14).

\section{Cálculo del Rendimiento Óptimo Sostenible (OSY)}

La Tabla 9 muestra los resultados del rendimiento óptimo sostenible del recurso anchoveta y sus correspondientes niveles de esfuerzo pesquero y biomasa de la zona norte-centro para el período 2004-2008, calculados según el modelo bioeconómico dinámico en tiempo continuo de Clark-Munro, con una tasa social de descuento de $\delta=10,596$ (Tabla 6).

El modelo de Clark-Munro, a diferencia del modelo de Gordon-Schaefer, considera en su formulación la variable tiempo, implícita en la aplicación de la tasa social de descuento que permite calcular y maximizar el valor presente de los flujos de retornos económicos netos (renta del recurso) futuros.

Tabla 9. Cálculo del Rendimiento Óptimo Sostenible

\begin{tabular}{c|c}
\hline Ecuación & Valor \\
\hline \hline Biomasa óptima ( $($ ) & \\
\hline$X^{*}=\frac{K}{4}\left[\left(\frac{c}{p q K}+1-\frac{\delta}{r}\right)+\sqrt{\left.\left(\frac{c}{p q K}+1-\frac{\delta}{r}\right)^{2}+\frac{8 c \delta}{p q K r}\right]}\right.$ & 22.596 .442 \\
\hline$H^{*}=r \cdot X^{*}\left(1-\frac{X^{*}}{K}\right)$ & 6.176 .636 \\
\hline Esfuerzo óptimo (m3-desembarque) & 11.128 .454 \\
$E^{*}=\frac{H^{*}}{q \cdot X^{*}}$ & \\
\hline
\end{tabular}


La estimación de la biomasa óptima $\left(X^{*}\right)$ es de singular importancia en el modelo de ClarkMunro, pues debe convertirse en el objetivo de la administración pesquera, manejando la captura como variable de control. Así, con una tasa social de descuento de $10,956 \%$, se calculó una biomasa óptima $\left(X^{*}\right)$ de 22.596 .442 toneladas, inferior en $2,62 \%$ a la biomasa del nivel de rendimiento máximo económico $\left(X_{M E Y}\right)$ de 23.205 .959 toneladas calculada mediante el modelo de Gordon-Schaefer, el cual no considera en su formulación una tasa social de descuento $(\delta)$. Como se mencionó anteriormente, la biomasa hidroacústica anual de anchoveta estimada por Imarpe durante el período 2004-2008, estuvo por debajo de 12,7 millones de toneladas.

La ecuación (35) del modelo de optimización de Clark-Munro, sugiere invertir en el recurso en caso que la biomasa óptima estimada resulte superior a la biomasa actual, a fin de alcanzar, tan rápido como sea posible, el nivel poblacional óptimo. En este caso, invertir en el recurso significa cerrar la zona de pesca completamente y suspender temporalmente la actividad pesquera $(h=$ 0), a fin de permitir la reconstrucción del recurso (Scott, 1955; Clark y Munro, 2017).

La captura óptima $\left(H^{*}\right)$ es de 6.176 .3636 toneladas, esto es, $4.4 \%$ mayor que la captura $\left(H_{M E Y}\right)$ de 5.916.558 toneladas, mientras que el esfuerzo óptimo $\left(E^{*}\right)$ fue de $11.128 .454 \mathrm{~m}^{3}$ desembarque, $7,2 \%$ mayor que el esfuerzo $\left(E_{M E Y}\right)$ de $10.379 .883 \mathrm{~m}^{3}$-desembarque.

Conviene anotar que, en general, los resultados de esfuerzo y biomasa de anchoveta en los niveles de MSY, MEY y OSY coinciden con el marco teórico explicitado en las Figuras 5 y 6.

La captura óptima $\left(H^{*}\right)$ y el esfuerzo óptimo $\left(E^{*}\right)$, permitirán calcular la renta óptima del recurso $\left(\pi^{*}\right)$.

\section{Cálculo de la Renta del Recurso Óptima}

La Tabla 10 muestra los resultados de la renta del recurso óptima $\left(\pi^{*}\right)$ de la zona norte-centro para el período 2004-2008, así como $H^{*}, E^{*}, X^{*}$ y el $C P U E^{*}$, calculados en base al modelo bioeconómico dinámico en tiempo continuo de Clark-Munro, aplicando diversos niveles de tasa social de descuento $(\delta)$. La renta del recurso óptima $\left(\pi^{\star}\right)$ se calcula a partir de la ecuación (23).

Tabla 10. Renta del Recurso Óptima a diversos niveles de tasa social de descuento

\begin{tabular}{|c|c|c|c|c|c|c|}
\hline Modelo & $\begin{array}{c}\delta \\
(\%)\end{array}$ & $\begin{array}{c}H^{*} \\
(t)\end{array}$ & $\begin{array}{c}E^{*} \\
(m 3- \\
\text { desembarque) }\end{array}$ & $\begin{array}{c}X^{*} \\
(t)\end{array}$ & $\begin{array}{c}\pi^{*} \\
(U S S)\end{array}$ & $\begin{array}{c}\text { CPUE* } \\
(\text { t/m3- } \\
\text { desembarque) }\end{array}$ \\
\hline \multirow{5}{*}{$\begin{array}{l}\text { Clark- } \\
\text { Munro }\end{array}$} & 0 & 5.916 .558 & 10.379 .883 & 23.205 .959 & 275.086 .669 & 0.570 \\
\hline & 5 & 6.045 .251 & 10.742 .238 & 22.910 .915 & 274.751 .432 & 0.563 \\
\hline & 10,59 & 6.176 .636 & 11.128 .454 & 22.596 .442 & 273.655 .962 & 0.555 \\
\hline & 20 & 6.370 .488 & 11.733 .561 & 22.103 .740 & 270.408 .078 & 0.543 \\
\hline & $\infty$ & 7.523 .436 & 20.759 .766 & 14.754 .234 & 0 & 0.362 \\
\hline
\end{tabular}

La Renta del Recurso Óptima $\left(\pi^{*}\right)$ estimada con una tasa social de descuento $\delta=10,59 \%$, es de US\$273.655.962. Dicho monto total se obtiene a partir de una biomasa óptima $\left(X^{\star}\right)$ de 22.596.442 toneladas, una captura óptima $\left(H^{*}\right)$ de 6.176.636 toneladas y un esfuerzo óptimo $\left(E^{\star}\right)$ de $11.128 .454 \mathrm{~m}^{3}$-desembarque.

Se observa, asimismo, que la biomasa óptima $\left(X^{*}\right)$, varía entre 23.205 .959 toneladas $(\delta=0)$ y 14.754.234 toneladas $(\delta=\infty)$. Se confirma que los valores obtenidos con la tasa social de descuento $\delta=0$ (es decir, sin tasa social de descuento), coinciden con los valores estimados para el $M E Y$, lo cual supone que el "modelo Gordon-Schaefer, implícitamente, asume que la tasa social de descuento, $\delta$, es: $\delta=0$ " (Clark y Munro, 2017) y que el modelo dinámico de ClarkMunro es una extensión del modelo estático de Gordon-Schaefer. 


\section{Cálculo del derecho de pesca}

Los resultados del cálculo de los derechos de pesca $(F F)^{14}$, se muestran en la Tabla 11. El FF se calculó mediante la ecuación (37) aplicada por Viet Thanh (2006) en su análisis bioeconómico de la pesquería del langostino en el Golfo de Tonkin, Vietnam, la cual se ilustra en la Figura 10. Y, los resultados se muestran en la Tabla 11.

$$
(p-F F) . H^{*}=c \cdot E^{*}
$$

por lo tanto:

$$
F F=p-\left(c . E^{*} / H^{*}\right)
$$

donde $p$ es el precio de la captura $\left(H^{*}\right)$ por tonelada en el desembarque y c es el costo unitario del esfuerzo óptimo $\left(E^{*}\right)$. En términos unitarios, el derecho de pesca estaría dado por la diferencia entre el precio de la captura y el costo del esfuerzo en el nivel del rendimiento óptimo sostenible (OSY).

Se consideró conveniente estimar los derechos de pesca para los mismos niveles de tasa social de descuento aplicados en el caso del cálculo de la renta del recurso óptima, a efectos de ver los cambios en función a la variación de la tasa de descuento.

Los datos de captura óptima $\left(H^{\star}\right)$ y esfuerzo óptimo $\left(E^{\star}\right)$, aplicados en la ecuación (36) corresponden a los mostrados en la Tabla 10; $y$, los datos de precio del recurso por tonelada y el costo unitario del esfuerzo pesquero son los que se muestran en la Tabla 6 , los cuales -como ya se mencionó- se asumen constantes para el período de pesca $2004-2008$, correspondiendo a precios y costos promedio de dicho período.

Figura 10. Derecho de pesca en el nivel de captura óptima y esfuerzo óptimo

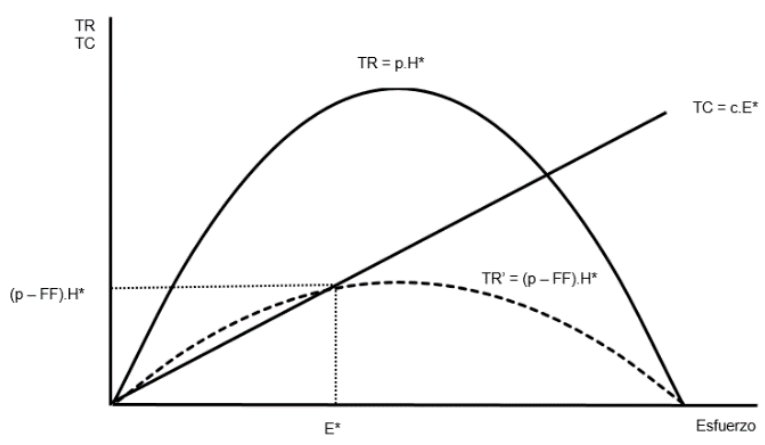

Tabla 11. Derecho de pesca a diversos niveles de tasa social de descuento

\begin{tabular}{c|c|c}
\hline \multirow{4}{*}{ Modelo } & $\begin{array}{c}\delta \\
(\%)\end{array}$ & $\begin{array}{c}\text { Derecho de Pesca } \\
\text { (FF) } \\
\text { (USS/t) }\end{array}$ \\
\hline \hline \multirow{4}{*}{ Clark- Munro } & 0 & 46,49 \\
\cline { 2 - 3 } & 5 & 45,45 \\
\cline { 2 - 3 } & 10,59 & 44,31 \\
\cline { 2 - 3 } & 20 & 42,45 \\
\cline { 2 - 3 } & $\infty$ & 0 \\
\hline
\end{tabular}

${ }^{14}(F F)=$ Fishing Fees 
El monto del derecho de pesca, calculado a la tasa social de descuento aplicada en proyectos de inversión pública ( $\delta=10,59 \%$ ) y bajo condiciones de optimización de la biomasa, de la captura y del esfuerzo pesquero, fue de US\$ 44,31/t (35\% del precio del recurso). Estudios han demostrado que los niveles históricos de renta podrían haber estado entre 10 y 60 por ciento de los ingresos brutos de la pesca (Campbell y Haynes, 1990).

\section{Implicancias de política}

El análisis bioeconómico del caso de la pesquería de la anchoveta peruana genera algunas implicancias de política pesquera relativas a dos aspectos fundamentales: el cálculo de los derechos de pesca y la gestión del recurso.

Con respecto al primer tema, queda en evidencia que existe base teórica suficiente para sustentar -desde una perspectiva científica- una nueva metodología para el cálculo de los derechos de pesca del recurso anchoveta, relativo a la aplicación del concepto de maximización de la renta del recurso, que incluye -únicamente- los costos derivados de la actividad extractiva o esfuerzo de pesca. Asimismo, la autoridad pesquera deberá elegir si, en su condición de propietario del recurso, toma la totalidad de la renta del recurso o sólo parte de ella. Esta decisión estará en función del análisis de los costos de administración de la pesquería y de las necesidades de reinversión en activos fijos del armador pesquero o en la diversificación de su inversión en la industria pesquera y su impacto en la generación de empleo.

Con relación al segundo tema, la gestión del recurso supone la inversión en el stock del recurso hasta alcanzar el nivel estimado de estado estacionario de la biomasa, usando la captura como variable de control. Invertir en el stock significa incorporar a la biomasa parte o todo el volumen del crecimiento natural del recurso, cuya captura se decide diferir para aumentar la cantidad disponible a usarse en fecha futura con un beneficio mayor y sostenido. Así, el administrador de la pesquería, deberá analizar diferentes escenarios que permitan alcanzar en el menor tiempo posible el nivel estimado de estado estacionario de la biomasa (Figura 7), sin dejar de considerar dentro del análisis los posibles efectos socioeconómicos de la medida a seleccionarse. Uno de los posibles escenarios a analizar sería la moratoria completa de la pesquería por un período determinado, el cual permitiría alcanzar más rápidamente el stock deseado, sin embargo esta medida draconiana pasa por evaluar la flexibilidad tanto el capital producido como el capital humano, es decir, "que ambos capitales puedan ser movidos con facilidad y sin costo significativo dentro o fuera de la pesquería" (Clark y Munro, 2017). Otro escenario sería la elección arbitraria de un volumen de captura anual. En todos los casos, resulta fundamental el plazo de recuperación del stock, que considera la captura anual autorizada y el saldo del crecimiento natural que se incorpora al stock al final del período anual, así como el cálculo del valor presente de los flujos netos anuales de las capturas. El valor presente de los ingresos netos anuales, permite comparar cada escenario y elegir el que resulte más rentable.

\section{CONCLUSIONES}

[1] El presente estudio confirma que la renta del recurso, estimada mediante el modelo bioeconómico de optimización dinámica en tiempo continuo de Clark y Munro (1975), puede constituirse en un fundamento económico válido para el cálculo de los derechos de pesca de la anchoveta peruana (Engraulis ringens). Los resultados fueron validados mediante el modelo bioeconómico estático de Gordon-Schaefer (1954), ampliamente usado en la economía pesquera.

[2] El derecho de pesca calculado fue de US $\$ 44,31 /$ para el período de acceso abierto regulado 2004-2008 (prereforma). No fue posible aplicar el modelo bioeconómico dinámico de ClarkMunro al período 2009-2017 correspondiente al régimen de cuotas individuales de pesca (reforma), debido a que la unidad de medida seleccionada del esfuerzo pesquero no resultó compatible con los fundamentos del marco teórico a la hora de aplicarse a la dinámica de pesca de dicho régimen, siendo necesario, por tanto, profundizar los estudios en esta materia. No obstante, dado que, el régimen de pesca del período 2004-2008 se considera menos eficiente que el régimen de cuotas individuales de pesca, y por tanto, este último más rentable que el primero, resulta razonable considerar el monto de derecho de pesca calculado como monto base de referencia y, de hecho, menor al que pudiera determinarse para el régimen de cuotas individuales de pesca. 
[3] Los parámetros biológicos, como la captura óptima (6.176.636 toneladas) y la biomasa óptima (22.596.442 toneladas), estimados por el modelo bioeconómico dinámico de ClarkMunro para el período 2004-2008, resultarían aplicables al período 2009-2017 del régimen de cuotas individuales de pesca, al tratarse del mismo stock poblacional, el cual durante dicho período muestra una captura promedio anual de 3,7 millones de toneladas. Por tanto, resultaría razonable invertir en el stock del recurso a fin de facilitar su recuperación y alcanzar el nivel de estado estacionario óptimo estimado de la biomasa para el período 2004-2008, y alcanzar un nivel de captura sostenible óptima ad infinitum. Los esquemas de recuperación del stock responden a un análisis socioeconómico de la pesquería, tal como se discutió en el acápite "Implicancias de política".

\section{FUENTES DE INFORMACIÓN}

Berachi, I. (2003). Bioeconomic analysis of artisanal marine fisheries of Tanzania (Mainland). MSc. Thesis in International Fisheries Management. University of Tromsø, Norway.

Beverton, R. J. H. y Holt, S. J. (1993). On the dynamics of exploited fish populations. Chapman \& Hall, London.

Bjørndal, T. y Bezabih, M. (2010). Resource Rents and Management Regimes: The Case of the Western Channel Sole Fishery. Marine Resource Economics, Volume 25, pp. 325-331.

Bonifaz, J. y Lama, R. (2013). Optimización Dinámica y Teoría Económica (1era. ed. corregida). Universidad del Pacífico, Centro de Investigación. Lima.

Campbell, D. y Haynes, J. (1990). Resource Rents in Fisheries. Australian Bureau of Agricultural and Resource Economics. Canberra.

Clark, C. W. (1990). Mathematical bioeconomics: the optimal management of renewable resources (2da. ed). New York: Wiley.

Clark, C. W. (2006). Fisheries bioeconomics: why is it so widely misunderstood? Population Ecology, 48:95-98.

Clark, C. W., Clarke, F. H. y Munro, G. (1979). The Optimal Management the Renewable Resources Stocks: Problems of Irreversible Investment. Econometrica 47(1):25-47.

Clark, C. W. y Munro, G. R. (2017). Capital Theory and the Economics of the Fisheries: Implications for Policy. Marine Resource Economics, volume 32, number 2.

Clark, C. W. y Munro, G. R. (1975). Economics of fishing and modern capital theory: a simplified approach. Journal of Environmental Economics and Management, 2, 92- 106.

Csirke, J. y Gumy, A. (1996). Análisis bioeconómico de la pesquería pelágica peruana dedicada a la producción de harina y aceite de pescado. Boletín Vol 15, № 2, Instituto del Mar del Perú (IMARPE), Callao, Perú.

Csirke, J. (1989a). Changes in the catchability coefficient in the Peruvian anchoveta (Engraulis ringens) fishery, p. 207-219. En: D. Pauly, P. Muck, J. Mendo and I. Tsukayama (eds.) The Peruvian upwelling ecosystem: dynamics and interactions. ICLARM Conference Proceedings 18, 438 p. Instituto del Mar del Perú (IMARPE), Callao, Perú; Deutsche Gesellschaft für Technische Zusammerarbeit (GTZ) GmbH, Eschbom, Federal Republic of Germany; and International Center for Living Aquatic Resources Management (ICLARM), Manila, Philippines.

Csirke, J. (1989b). Introducción a la dinámica de poblaciones de peces. FAO, Documento Técnico de Pesca, (192):82 p.

De La Puente, O., Sueiro, J., Heck, C., Soldi, G. y De La Puente, S. (2011). La pesquería peruana de Anchoveta. Centro para la Sostenibilidad Ambiental, Universidad Peruana Cayetano Heredia.

FAO (2018). Food Outlook. Biannual Report on Global Food Markets. Roma.

Flaaten, O. (2014). Fisheries Economics and Management. Norwegian College of Fishery Science University of Tromsø. Norway.

Galarza, E. y Collado, N. (2013). Los derechos de pesca: el caso de la pesquería de anchoveta peruana. Apuntes: Revista de Ciencias Sociales, Vol. XL, No 73, 7-42. Centro de Investigación de la Universidad del Pacífico.

Gordon, H. S. (1954). The Economic Theory of Common Property Resources: The Fishery. Journal of Political Economy 62, No. 2: 124-142.

Ministerio de Economía y Finanzas (2012). Actualización de la tasa social de descuento. Recuperado de https://www.mef.gob.pe/contenidos/inv publica/docs/estudios documentos/estudios/Actuali zacion TSD Junio 2012.pdf 
Munro, G. (2010). The Economics of Overcapacity and the Management of Capture Fishery Resources: A Review. International Review of Environmental and Resource Economics 4(2):93-122.

Munro, G. y Sumaila, U. (2015). On the Contributions of Colin Clark to Fisheries Economics. Environmental and Resource Economics 61(1):1-17.

Oliveros, D. (2009). Modelo de control viable para el manejo sostenible del stock de anchoveta peruana (Engraulis ringens). Tesis para optar el título de Biólogo. Facultad de Ciencias, Universidad Nacional Agraria La Molina. 66 pp.

Paredes, C. (2012). Eficiencia y equidad en la pesca peruana: la reforma y los derechos de pesca. Lima, Instituto del Perú, Universidad de San Martín de Porres.

Pontryagin, L., Boltyanskii, V., Gramkrelidze, R. y Mishchenko, E. (1962). "The Mathematical Theory of Optimal Processes". Wiley, New York.

Rodgers, T. y Webster, S. (2007). Resource rent mechanisms in Australian primary industries: some observations and issues. Paper presented at the 51st Annual Conference 2007 of the Australian Agricultural and Resource Economics Society Conference. Australia.

Schaefer, M. B. (1954). Some Aspects of the Dynamics of Populations Important to the Management of the Commercial Marine Fisheries. Inter-American Tropical Tuna Commission. $<<$ Bulletin, 1(2), 27-51.

Schaefer, M. B. (1957): Some considerations of population dynamics and economics in relation to the management of marine fisheries. Journal of the Fisheries Research Board of Canada, 14, 669-681.

Scott, A. (1955). The Fishery: The Objetives of Sole Ownership. Journal of Political Economy, 63(2), University of Chicago Press. pp. 116 - 124.

Viet Thanh, N. (2006). Bioeconomic analysis of the shrimp trawl fishery in the Tonkin Gulf, Vietnam. MSc. Thesis. University of Tromsø, Norway.

World Bank Group (2017). The Sunken Billions Revisited: Progress and Challenges in Global Marine Fisheries. Washington, DC: World Bank. Environment and Sustainable Development series. doi:10.1596/978-1-4648-0919-4. License: Creative Commons Attribution CC BY 3.0 I 